\title{
INCREMENTAL MOMENTS AND HÖLDER EXPONENTS OF MULTIFRACTIONAL MULTISTABLE PROCESSES
}

\author{
Ronan Le Guével ${ }^{1}$ AND JACQues LÉvy VÉHeL ${ }^{2}$
}

\begin{abstract}
Multistable processes, that is, processes which are, at each "time", tangent to a stable process, but where the index of stability varies along the path, have been recently introduced as models for phenomena where the intensity of jumps is non constant. In this work, we give further results on (multifractional) multistable processes related to their local structure. We show that, under certain conditions, the incremental moments display a scaling behaviour, and that the pointwise Hölder exponent is, as expected, related to the local stability index. We compute the precise value of the almost sure Hölder exponent in the case of the multistable Lévy motion, which turns out to reveal an interesting phenomenon.
\end{abstract}

Mathematics Subject Classification. 60G17, 60G18, 60G22, 60G52.

Received September 2, 2010. Revised March 19, 2011.

\section{INTRODUCTION}

Multistable processes are stochastic processes which are "locally stable", but where the index of stability varies with "time". To be more precise, we need to recall the definition of a localisable process $[4,5]: Y=\{Y(t): t \in \mathbf{R}\}$ is said to be $h$-localisable at $u$ if there exists an $h \in \mathbf{R}$ and a non-trivial (i.e. finite and non-zero) limiting process $Y_{u}^{\prime}$ such that

$$
\lim _{r \rightarrow 0^{+}} \frac{Y(u+r t)-Y(u)}{r^{h}}=Y_{u}^{\prime}(t)
$$

(Note $Y_{u}^{\prime}$ may and in general will vary with $u$ ). When the limit exits, $Y_{u}^{\prime}=\left\{Y_{u}^{\prime}(t): t \in \mathbf{R}\right\}$ is termed the local form or tangent process of $Y$ at $u$. The limit (1.1) may be taken in mainly two ways: convergence in finite dimensional distributions, or in distribution when the paths of the process are continuous or càdlàg (in which case the process is called strongly $h$-localisable).

A classical example of a strongly localisable process is multifractional Brownian motion $Y$ [1,2, 10, 18] which "looks like" index- $h(u)$ fractional Brownian motion close to time $u$ but where $h(u)$ varies, that is

$$
\lim _{r \rightarrow 0^{+}} \frac{Y(u+r t)-Y(u)}{r^{h}}=B_{h(u)}(t)
$$

Keywords and phrases. Localisable processes, multistable processes, multifractional processes, pointwise Hölder regularity.

1 Université Paris VI, Laboratoire de Probabilités et Modèles Aléatoires 4 place Jussieu, 75252 Paris Cedex 05, France. ronan.leguevel@upmc.fr

2 Regularity team, INRIA Saclay, Parc Orsay Université 4 rue Jacques Monod, Bat P, 91893 Orsay Cedex, France.

jacques.levy-vehel@inria.fr 
where $B_{h}$ is index- $h$ fractional Brownian motion. A generalization of $\mathrm{mBm}$, where the Gaussian measure is replaced by an $\alpha$-stable one, leads to multifractional stable processes, where the local form is an $h(u)$-selfsimilar linear $\alpha$-stable motion [22,23].

Multifractional multistable processes provide a further step of generalization: they are localisable processes such that the tangent process is again an $\alpha$-stable random process, but where $\alpha$ now varies with time. Multifractional multistable processes were constructed in $[6-8,13]$ using respectively moving averages, sums over Poisson processes, the Ferguson-Klass-LePage series representation, and multistable measures. Section 3.3 below provides several specific examples of such processes.

The aim of this work is twofold:

1. We show that, for a large class of (multifractional) multistable processes, a precise estimate for the incremental moments holds. More precisely, we prove in Section 3.1 that there exists a natural scaling relation for $\mathrm{E}\left[|Y(t+\varepsilon)-Y(t)|^{\eta}\right]$ and $\varepsilon$ small. This class includes (multifractional) multistable processes considered in $[6,13]$, in particular Lévy multistable motions and linear multistable multifractional motions. It also include certain moving average multistable processes such as the reverse multistable Ornstein-Uhlenbeck process of [8].

2. We then study the pointwise Hölder regularity of (multifractional) multistable processes. For the same class as above, we obtain an almost sure upper bound for this exponent. In the case of the Lévy multistable motion, we are able to compute its exact value. An interesting phenomenon occurs: when the functional parameter $\alpha$ is smooth, not surprisingly, the Hölder exponent is equal, at each point, almost surely, to the localisability index. However, when $\alpha$ is smaller than one and sufficiently irregular, the regularity of the process is governed by the one of the function $\alpha$ : their Hölder exponents coincide almost surely. Note that a uniform statement, i.e. a statement like "almost surely, at each point", cannot hold true in general. Indeed, it already fails for the case of a Lévy stable motion. The right frame in this respect is multifractal analysis, and results in this direction will be presented in a forthcoming work.

The remainder of this work is organized as follows. In the next section, we recall the definition of multistable processes based on the Ferguson-Klass-LePage series representation used in [13] (this defines processes which are equal in distribution to the ones obtained in [6] through sums over Poisson processes). Our main results on incremental moments and upper bound for the pointwise Hölder exponents are described in Sections 3.1-3.3 applies these findings to linear multistable multifractional motion. In Section 3.4, we state the result giving the exact value of the pointwise Hölder regularity of Lévy multistable motion. An exemple of a moving average multistable process (reverse multistable Ornstein-Uhlenbeck) is the topic of Section 3.5. In Section 4, we give intermediate results, some of which being of independent interest, which are used in the proofs of the main statements. Section 5 gathers technical results followed by the proofs of the statements related with the incremental moments and upper bounds on the exponents. Section 6 contains the computation of the exponent for the multistable Lévy motion. Finally, Section 7 gives a list of the various technical conditions on multistable processes required by our approach so that their incremental moments and Hölder exponents may be estimated.

\section{Multistable processes}

We now define multistable processes using the Ferguson-Klass-LePage series representation. These are defined as "diagonals" of random fields that are described below. In the sequel, $(E, \mathcal{E}, m)$ will be a measure space, and $U$ an open interval of $\mathbf{R}$. We will assume that $m$ is a finite or $\sigma$-finite measure. Let $\alpha$ be a $C^{1}$ function defined on $U$ and ranging in $[c, d] \subset(0,2)$. Let $f(t, u,$.$) be a family of functions such that, for all (t, u) \in U^{2}$, $f(t, u,.) \in \mathcal{F}_{\alpha(u)}(E, \mathcal{E}, m)$, where:

$$
\mathcal{F}_{\alpha} \equiv \mathcal{F}_{\alpha}(E, \mathcal{E}, m)=\left\{f: f \text { is measurable and }\|f\|_{\alpha}<\infty\right\},
$$


and \|\|$_{\alpha}$ is the quasinorm (or norm if $1<\alpha \leq 2$ ) given by

$$
\|f\|_{\alpha}=\left\{\begin{array}{cc}
\left(\int_{E}|f(x)|^{\alpha} m(\mathrm{~d} x)\right)^{1 / \alpha} & (\alpha \neq 1) \\
\int_{E}|f(x)| m(\mathrm{~d} x)+\int_{E}|f(x) \beta(x) \ln | f(x) \| m(\mathrm{~d} x) & (\alpha=1) .
\end{array}\right.
$$

By assumption on $m$, there exists $r: E \rightarrow \mathbb{R}_{+}$such that $\hat{m}(\mathrm{~d} x)=\frac{1}{r(x)} m(\mathrm{~d} x)$ is a probability measure (see, e.g., [21], Prop. 3.11.3). When $m$ is a finite measure, we always take $r(x) \equiv m(E)$.

The following notations are used throughout in the sequel: $\left(\Gamma_{i}\right)_{i \geq 1}$ will be a sequence of arrival times of a Poisson process with unit arrival time. $\left(V_{i}\right)_{i \geq 1}$ will denote a sequence of i.i.d. random variables with distribution $\hat{m}$ on E. Finally, $\left(\gamma_{i}\right)_{i \geq 1}$ will be a sequence of i.i.d. random variables with distribution $P\left(\gamma_{i}=1\right)=P\left(\gamma_{i}=-1\right)=1 / 2$. The three sequences $\left(\Gamma_{i}\right)_{i \geq 1},\left(V_{i}\right)_{i \geq 1}$, and $\left(\gamma_{i}\right)_{i \geq 1}$ are independent.

As in [13], we will consider the following random field:

$$
X(t, u)=C_{\alpha(u)}^{1 / \alpha(u)} \sum_{i=1}^{\infty} \gamma_{i} \Gamma_{i}^{-1 / \alpha(u)} r\left(V_{i}\right)^{1 / \alpha(u)} f\left(t, u, V_{i}\right),
$$

where $C_{\eta}=\left(\int_{0}^{\infty} x^{-\eta} \sin (x) \mathrm{d} x\right)^{-1}$.

Note that when the function $\alpha$ is constant, then (2.4) is just the Ferguson-Klass-LePage series representation of a stable random variable (see [3,9,14,15,20] and [21], Thm. 3.10.1, for specific properties of this representation).

\section{Multistable processes}

Multistable processes are obtained by taking diagonals on $X$, i.e. setting $Y(t)=X(t, t)$ : as shown in Theorems 3.3 and 4.5 of [13], provided both $X$ and $f$ fulfill certain conditions, $Y$ is a localisable process whose local form is a stable process. In the sequel, we obtain, under some assumptions (which imply that $Y$ is indeed localisable), estimates on the incremental moments and the pointwise Hölder regularity of $Y$. We will always assume that $t \mapsto X(t, u)$ is localisable at any $u$ with exponent $h(u) \in\left(h_{-}, h_{+}\right) \subset(0,1)$. The local form is denoted $X_{u}^{\prime}(t, u)$. We assume in addition that $u \mapsto h(u)$ is a $C^{1}$ function.

\section{MAin RESUlts}

The following two theorems apply to a diagonal process $Y$ defined from the field $X$ given by (2.4). For convenience, the conditions required on $X$ and the kernel $f$ that appears in (2.4) are gathered in Section 7 .

\subsection{Moments of multistable processes}

Theorem 3.1. Let $t \in \mathbf{R}$ and $U$ be an open interval of $\mathbf{R}$ with $t \in U$. Let $\eta \in(0, c)$. Suppose that $f$ satisfies (R1), (M1), (M2), (M3) and (H2). Then, when $\varepsilon$ tends to 0 ,

$$
\mathrm{E}\left[|Y(t+\varepsilon)-Y(t)|^{\eta}\right] \sim \varepsilon^{\eta h(t)} \mathrm{E}\left[\left|Y_{t}^{\prime}(1)\right|^{\eta}\right] .
$$

$\left(f(x) \sim g(x)\right.$ when $x \rightarrow 0$ means that $\left.\lim _{x \rightarrow 0} f(x) / g(x)=1\right)$.

Proof. See Section 5.

Remark: Under the conditions listed in the theorem, Theorems 3.3 and 4.5 of [13] imply that $Y$ is $h(t)$-localisable at $t$. 


\subsection{Pointwise Hölder exponent of multistable processes}

Let $\mathcal{H}_{t}(\omega)=\sup \left\{\gamma: \lim _{r \rightarrow 0} \frac{|Y(t+r, \omega)-Y(t, \omega)|}{|r|^{\gamma}}=0\right\}$ denote the Hölder exponent of the (non-differentiable) process $Y$ at $t$.

Theorem 3.2 (upper bound). Suppose that there exists a function h defined on U such that (M1), (M2), (M3), (M4), (M5), (M6) and (M7) hold. Assuming (R1), (H1), (H3), (H4) and (H5), one has, for all t, almost surely:

$$
\mathcal{H}_{t} \leq h(t) .
$$

Proof. See Section 5.

\subsection{Example: linear multistable multifractional motion}

In this section, we apply the results above to the "multistable version" of a classical process known as linear stable multifractional motion, which is itself a extension of linear stable fractional motion. In the sequel, $M$ will always denote a symmetric $\alpha$-stable $(0<\alpha<2)$ random measure on $\mathbf{R}$ with control measure Lebesgue measure $\mathcal{L}$. The linear stable fractional motion is defined as follows [21]:

$$
L_{\alpha, H, b^{+}, b^{-}}(t)=\int_{-\infty}^{\infty} f_{\alpha, H}\left(b^{+}, b^{-}, t, x\right) M(\mathrm{~d} x)
$$

where $t \in \mathbb{R}, H \in(0,1), b^{+}, b^{-} \in \mathbb{R}$, and

$$
\begin{aligned}
f_{\alpha, H}\left(b^{+}, b^{-}, t, x\right)= & b^{+}\left((t-x)_{+}^{H-1 / \alpha}-(-x)_{+}^{H-1 / \alpha}\right) \\
& +b^{-}\left((t-x)_{-}^{H-1 / \alpha}-(-x)_{-}^{H-1 / \alpha}\right),
\end{aligned}
$$

where $(x)_{+}=\max \{0, x\}$ and $(x)_{-}=-(-x)_{+}$. When $b^{+}=b^{-}=1$, this process is called well-balanced linear fractional $\alpha$-stable motion and denoted $L_{\alpha, H}$.

The localisability of linear fractional $\alpha$-stable motion simply stems from the fact that it is $1 / \alpha$-self-similar with stationary increments [5].

The multifractional multistable version of this process was defined in $[6,13]$ (note that the choice of $\hat{m}$ is arbitrary as long as it fulfills the required condition, and is made purely for convenience). Its incremental moments and regularity are described by the following theorems:

Theorem 3.3 (linear multistable multifractional motion). Let $\alpha: \mathbf{R} \rightarrow[c, d] \subset(0,2)$ and $H: \mathbf{R} \rightarrow(0,1)$ be continuously differentiable. Let $\left(\Gamma_{i}\right)_{i \geq 1},\left(V_{i}\right)_{i \geq 1}$ and $\left(\gamma_{i}\right)_{i \geq 1}$ be as described in Section 2, where we take the distribution of $\left(V_{i}\right)_{i \geq 1}$ to be $\hat{m}(\mathrm{~d} x)=\frac{3}{\pi^{2}} \sum_{j=1}^{+\infty} j^{-2} \mathbf{1}_{[-j,-j+1[\cup[j-1, j[}(x) \mathrm{d} x$ on $\mathbf{R}$. Define:

$$
X(t, u)=C_{\alpha(u)}^{1 / \alpha(u)} \sum_{i, j=1}^{\infty}\left(\frac{\pi^{2} j^{2}}{3}\right)^{1 / \alpha(u)} \gamma_{i} \Gamma_{i}^{-1 / \alpha(u)}\left(\left|t-V_{i}\right|^{H(u)-1 / \alpha(u)}-\left|V_{i}\right|^{H(u)-1 / \alpha(u)}\right) \mathbf{1}_{[-j,-j+1[\cup[j-1, j[}\left(V_{i}\right)
$$

and the linear multistable multifractional motion

$$
Y(t)=X(t, t) .
$$

Then for all $t \in \mathbf{R}$ and $\eta<c$, when $\varepsilon$ tends to 0 ,

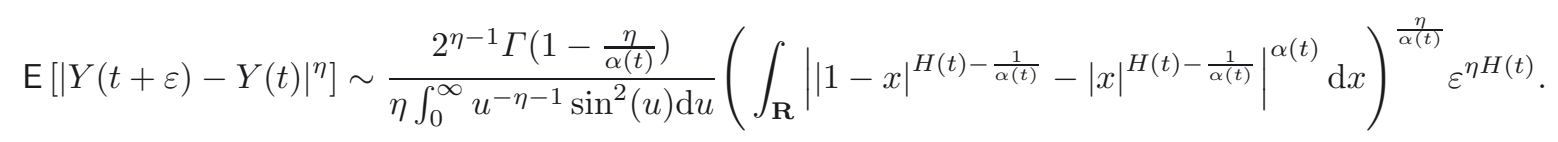

Proof. See Section 5. 
Theorem 3.4. Let $Y$ be the linear multistable multifractional motion defined on $\mathbf{R}$ with $H-\frac{1}{\alpha}$ a non-negative function. For all $t \in \mathbf{R}$, almost surely,

$$
\mathcal{H}_{t} \leq H(t)
$$

Proof. See Section 5.

\subsection{Example: Lévy multistable motion}

In the case of the Lévy multistable motion, we are able to provide a more precise result, to the effect that, at each point, the exact almost sure value of the Hölder exponent is known. Let us first recall some definitions. With $M$ again denoting a symmetric $\alpha$-stable $(0<\alpha<2)$ random measure on $\mathbf{R}$ with control measure Lebesgue measure $\mathcal{L}$, we write

$$
L_{\alpha}(t):=\int_{0}^{t} M(\mathrm{~d} z)
$$

for $\alpha$-stable Lévy motion.

The localisability of Lévy motion is a consequence of the fact that it is $1 / \alpha$-self-similar with stationary increments [5]. Its multistable version and incremental moments are described in the following theorem:

Theorem 3.5 (symmetric multistable Lévy motion). Let $\alpha:[0,1] \rightarrow[c, d] \subset(1,2)$ be continuously differentiable. Let $\left(\Gamma_{i}\right)_{i \geq 1},\left(V_{i}\right)_{i \geq 1}$ and $\left(\gamma_{i}\right)_{i \geq 1}$ be as described in Section 2, where we take the distribution of $\left(V_{i}\right)_{i \geq 1}$ to be $\hat{m}(\mathrm{~d} x)=\mathrm{d} x$ on $[0,1]$. Define

$$
X(t, u)=C_{\alpha(u)}^{1 / \alpha(u)} \sum_{i=1}^{\infty} \gamma_{i} \Gamma_{i}^{-1 / \alpha(u)} \mathbf{1}_{[0, t]}\left(V_{i}\right)
$$

and the symmetric multistable Lévy motion

$$
Y(t)=X(t, t) .
$$

Then for all $t \in(0,1)$ and $\eta<c$, when $\varepsilon$ tends to 0 ,

$$
\mathrm{E}\left[|Y(t+\varepsilon)-Y(t)|^{\eta}\right] \sim \frac{2^{\eta-1} \Gamma\left(1-\frac{\eta}{\alpha(t)}\right)}{\eta \int_{0}^{\infty} u^{-\eta-1} \sin ^{2}(u) \mathrm{d} u} \varepsilon^{\frac{\eta}{\alpha(t)}} .
$$

Proof. See Section 5 .

Theorem 3.6. Let $Y$ be the symmetric multistable Lévy motion defined on $[0,1]$ with $\alpha:[0,1] \rightarrow[c, d] \subset(0,2)$. For all $t \in(0,1)$, almost surely,

$$
\mathcal{H}_{t} \leq \frac{1}{\alpha(t)}
$$

Proof. See Section 5.

Theorem 3.7. Let $u \in U \subset(0,1)$.

1. If $0<\alpha(u)<1$, almost surely,

$$
\mathcal{H}_{u}=\min \left(\frac{1}{\alpha(u)}, \mathcal{H}_{u}^{\alpha}\right) .
$$

provided $\frac{1}{\alpha(u)} \neq \mathcal{H}_{u}^{\alpha}$, where $\mathcal{H}_{u}^{\alpha}$ denotes the Hölder exponent of $\alpha$ at $u$.

2. If $1 \leq \alpha(u)<2$, and $\alpha$ is $\mathcal{C}^{1}$, almost surely,

$$
\mathcal{H}_{u}=\frac{1}{\alpha(u)}
$$

Proof. See Section 6. 
Thus, in the case $0<\alpha(u)<1$, the regularity of multistable Lévy motion is the smallest number between $\frac{1}{\alpha(u)}$ and the regularity of the function $\alpha$ at $u$. This is very similar to the case of multifractional Brownian motion, where the Hölder exponent is the minimum between the functional parameter $h$ and its regularity [10,11]. We conjecture that the same result holds when $\alpha \geq 1$.

\subsection{Example: reverse multistable Ornstein-Uhlenbeck}

We consider in this section an exemple of a different nature, since the multistable process that we deal with is obtained by generalizing a moving average process rather than a self-similar one, as was the case of Lévy motion and linear fractional stable motion. This shows that conditions of Section 7 required for our results to hold, although somewhat numerous and technical, are indeed natural in our frame.

Let $\lambda>0$ and $1<\alpha \leq 2$ and let $M$ be an $\alpha$-stable measure on $\mathbf{R}$ with control measure $\mathcal{L}$. The stationary process

$$
Y(t)=\int_{t}^{\infty} \exp (-\lambda(x-t)) M(\mathrm{~d} x) \quad(t \in \mathbf{R})
$$

is called reverse Ornstein-Uhlenbeck process. By Proposition 2.2 of [8], it has a version in $D(\mathbf{R})$ that is $1 / \alpha-$ localisable at all $u \in \mathbf{R}$ with $Y_{u}^{\prime}=L_{\alpha}$.

A multistable version is obtained by taking

$$
r(x)=\sum_{j=1}^{+\infty} 2^{j+1} \mathbf{1}_{[-j,-j+1[\cup[j-1, j[}(x)
$$

and

$$
f(t, w, x)=\mathrm{e}^{-\lambda(x-t)} \mathbf{1}_{[t,+\infty)}(x)
$$

in (2.4) and considering as usual the diagonal process. Using the results of [13], it is easy to prove that $Y$ is $1 / \alpha(u)$-localisable at all $u \in \mathbf{R}$ with local form $Y_{u}^{\prime}=L_{\alpha(u)}$. We then have:

Theorem 3.8 (reverse Ornstein-Uhlenbeck multistable process). Let $\alpha: \mathbf{R} \rightarrow[c, d] \subset(1,2)$. Let $X(t, u)$ be the random field of (2.4) with $r$ given by (3.7) and $f$ given by (3.8). Define the reverse Ornstein-Uhlenbeck multistable process as

$$
Y(t)=X(t, t) .
$$

Then Theorems 3.1 and 3.2 applies to $Y$ at all $t \in \mathbf{R}$.

Proof. See Section 5.

\section{INTERMEDIATE RESULTS}

Let $\varphi_{X}$ denote the characteristic function of the random variable $X$. We first state the following almost obvious fact, which will be used in the proof of Theorem 3.2:

Proposition 4.9. Assume that for a given $t \in \mathbf{R}$ there exists $\varepsilon_{0}>0$ such that

$$
\sup _{t \in U} \sup _{r \in\left(0, \varepsilon_{0}\right)} \int_{0}^{+\infty}\left|\varphi_{\frac{Y(t+r)-Y(t)}{r^{h(t)}}}(v)\right| \mathrm{d} v<+\infty
$$

where $Y$ is a symmetrical process. Then there exists $K>0$ such that for all $t \in U$, for all $r \in\left(0, \varepsilon_{0}\right)$ and all $x>0$,

$$
\mathrm{P}(|Y(t+r)-Y(t)|<x) \leq K \frac{x}{r^{h(t)}} .
$$


Proof. This is a straightforward consequence of the inversion formula. Let $x>0$ and $0<r<\varepsilon_{0}$. Since $Y$ is a symmetrical process, $\varphi_{Y(t+r)-Y(t)}$ is an even function and

$$
\begin{aligned}
\mathrm{P}(|Y(t+r)-Y(t)|<x) & =\frac{1}{\pi}\left|\int_{0}^{+\infty} \varphi_{Y(t+r)-Y(t)}\left(\frac{v}{r^{h(t)}}\right) \sin \left(\frac{v x}{r^{h(t)}}\right) \frac{\mathrm{d} v}{v}\right| \\
& \leq \frac{1}{\pi} \frac{x}{r^{h(t)}} \sup _{r \in B\left(0, \varepsilon_{0}\right)} \int_{0}^{+\infty} \mid \frac{\varphi_{\frac{Y(t+r)-Y(t)}{r^{h(t)}}}(v) \mid \mathrm{d} v}{} \\
& \leq K \frac{x}{r^{h(t)}} .
\end{aligned}
$$

The next proposition will also be used in the proof of Theorem 3.2:

Proposition 4.10. Suppose that there exists a function h defined on $U$ such that (H1), (H3), (H4), and (H5) hold. Assuming (R1), (M4), (M5), (M6), and (M7) one has:

$$
\sup _{t \in U} \sup _{r \in\left(0, \varepsilon_{0}\right)} \int_{0}^{+\infty}\left|\varphi_{\frac{Y(t+r)-Y(t)}{r^{h(t)}}}(v)\right| \mathrm{d} v<+\infty .
$$

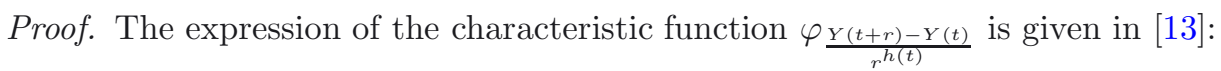

$$
\varphi_{\frac{Y(t+r)-Y(t)}{r^{h(t)}}}(v)=\exp \left(-2 \int_{\mathbf{R}} \int_{0}^{+\infty} \sin ^{2}\left(\frac{v C_{\alpha(t+r)}^{1 / \alpha(t+r)} f(t+r, t+r, x)}{2 r^{h(t)} y^{1 / \alpha(t+r)}}-\frac{v C_{\alpha(t)}^{1 / \alpha(t)} f(t, t, x)}{2 r^{h(t)} y^{1 / \alpha(t)}}\right) \mathrm{d} y m(\mathrm{~d} x)\right) .
$$

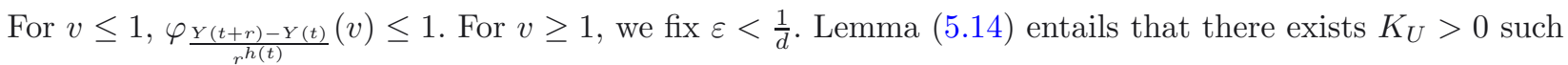
that

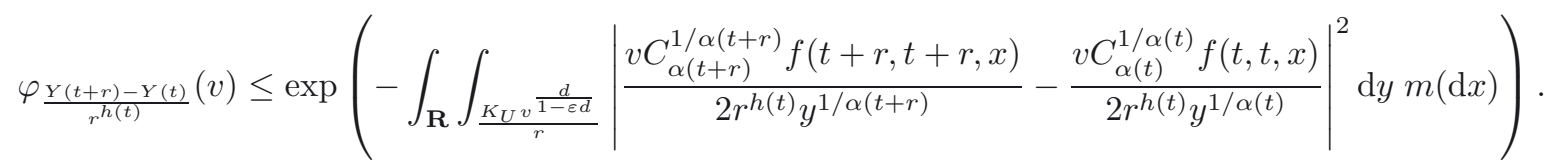

Let

$$
N(v, t, r)=\int_{\mathbf{R}} \int_{\frac{K_{U} v^{1}}{r} \frac{d}{r}}\left|\frac{v C_{\alpha(t+r)}^{1 / \alpha(t+r)} f(t+r, t+r, x)}{2 r^{h(t)} y^{1 / \alpha(t+r)}}-\frac{v C_{\alpha(t)}^{1 / \alpha(t)} f(t, t, x)}{2 r^{h(t)} y^{1 / \alpha(t)}}\right|^{2} \mathrm{~d} y m(\mathrm{~d} x) .
$$

Using Lemma 5.15, there exist $K_{U}>0$ and $\varepsilon_{0}>0$ such that for all $v \geq 1$,

$$
N(v, t, r) \geq K_{U} v^{2+\frac{d}{1-\varepsilon d}\left(1-\frac{2}{c}\right)} .
$$

The inequality becomes

$$
\varphi_{\frac{Y(t+r)-Y(t)}{r^{h(t)}}}(v) \leq \exp \left(-K_{U} v^{2+\frac{d}{1-\varepsilon d}\left(1-\frac{2}{c}\right)}\right),
$$

and

$$
\begin{aligned}
\int_{0}^{+\infty} \varphi_{\frac{Y(t+r)-Y(t)}{r^{h(t)}}}(v) \mathrm{d} v & \leq 1+\int_{1}^{\infty} \exp \left(-K_{U} v^{2+\frac{d}{1-\varepsilon d}\left(1-\frac{2}{c}\right)}\right) \mathrm{d} v \\
& <+\infty .
\end{aligned}
$$

We now consider multistable processes. The next proposition will be used in the proof of Theorem 3.1. 
Proposition 4.11. Assuming (R1), (M1), (M2) and (M3), there exists $K_{U}>0$ such that for all $u \in U, v \in U$ and $x>0$,

$$
\mathbf{P}(|X(v, v)-X(v, u)|>x) \leq K_{U}\left(\frac{|v-u|^{d}}{x^{d}}\left(1+\left|\log \frac{|v-u|}{x}\right|^{d}\right)+\frac{|v-u|^{c}}{x^{c}}\left(1+\left|\log \frac{|v-u|}{x}\right|\right)\right) .
$$

Proof. See Section 5.

\section{Proofs And technical Results}

\subsection{Proof of Proposition 4.11}

First case: $r(x) \equiv 1$.

We proceed as in [13]. Note that condition (M1) implies that there exists $\delta^{\prime} \in\left(\frac{d}{c}-1, \delta\right)$ such that:

$$
\sup _{t \in U} \int_{\mathbf{R}}\left[\sup _{w \in U}\left[|f(t, w, x) \log | f(t, w, x)||^{\alpha(w)}\right]\right]^{1+\delta^{\prime}} m(\mathrm{~d} x)<\infty .
$$

Since (M1) is true with $\delta^{\prime}$ in place of $\delta$, we write in the sequel $\delta$ for $\delta^{\prime}$. The function $u \mapsto C_{\alpha(u)}^{1 / \alpha(u)}$ is a $C^{1}$ function since $\alpha(u)$ ranges in $[c, d] \subset(0,2)$. We shall denote $a(u)=(m(E))^{1 / \alpha(u)} C_{\alpha(u)}^{1 / \alpha(u)}$. The function $a$ is thus also $C^{1}$. Let $(u, v) \in U^{2}$.We estimate:

$$
X(v, v)-X(v, u)=\sum_{i=1}^{\infty} \gamma_{i}\left(\Phi_{i}(v)-\Phi_{i}(u)\right)+\sum_{i=1}^{\infty} \gamma_{i}\left(\Psi_{i}(v)-\Psi_{i}(u)\right)
$$

where

$$
\Phi_{i}(u)=a(u) i^{-1 / \alpha(u)} f\left(v, u, V_{i}\right)
$$

and

$$
\Psi_{i}(u)=a(u)\left(\Gamma_{i}^{-1 / \alpha(u)}-i^{-1 / \alpha(u)}\right) f\left(v, u, V_{i}\right)
$$

Thanks to the assumptions on $a$ and $f, \Phi_{i}$ and $\Psi_{i}$ are differentiable and one computes:

$$
\Phi_{i}^{\prime}(u)=a^{\prime}(u) i^{-1 / \alpha(u)} f\left(v, u, V_{i}\right)+a(u) i^{-1 / \alpha(u)} f_{u}^{\prime}\left(v, u, V_{i}\right)+a(u) \frac{\alpha^{\prime}(u)}{\alpha(u)^{2}} \log (i) i^{-1 / \alpha(u)} f\left(v, u, V_{i}\right),
$$

and

$$
\begin{aligned}
\Psi_{i}^{\prime}(u)= & a^{\prime}(u)\left(\Gamma_{i}^{-1 / \alpha(u)}-i^{-1 / \alpha(u)}\right) f\left(v, u, V_{i}\right)+a(u)\left(\Gamma_{i}^{-1 / \alpha(u)}-i^{-1 / \alpha(u)}\right) f_{u}^{\prime}\left(v, u, V_{i}\right) \\
& +a(u) \frac{\alpha^{\prime}(u)}{\alpha(u)^{2}}\left(\log \left(\Gamma_{i}\right) \Gamma_{i}^{-1 / \alpha(u)}-\log (i) i^{-1 / \alpha(u)}\right) f\left(v, u, V_{i}\right) .
\end{aligned}
$$

Using the mean value theorem, there exists a sequence of independent random numbers $w_{i} \in(u, v)$ (or $(v, u)$ ) and a sequence of random numbers $x_{i} \in(u, v)$ (or $(v, u)$ ) such that:

$$
X(v, u)-X(v, v)=(u-v) \sum_{i=1}^{\infty}\left(Z_{i}^{1}+Z_{i}^{2}+Z_{i}^{3}\right)+(u-v) \sum_{i=1}^{\infty}\left(Y_{i}^{1}+Y_{i}^{2}+Y_{i}^{3}\right)
$$


where

$$
\begin{gathered}
Z_{i}^{1}=\gamma_{i} a^{\prime}\left(w_{i}\right) i^{-1 / \alpha\left(w_{i}\right)} f\left(v, w_{i}, V_{i}\right) \\
Z_{i}^{2}=\gamma_{i} a\left(w_{i}\right) i^{-1 / \alpha\left(w_{i}\right)} f_{u}^{\prime}\left(v, w_{i}, V_{i}\right) \\
Z_{i}^{3}=\gamma_{i} a\left(w_{i}\right) \frac{\alpha^{\prime}\left(w_{i}\right)}{\alpha\left(w_{i}\right)^{2}} \log (i) i^{-1 / \alpha\left(w_{i}\right)} f\left(v, w_{i}, V_{i}\right) \\
Y_{i}^{1}=\gamma_{i} a^{\prime}\left(x_{i}\right)\left(\Gamma_{i}^{-1 / \alpha\left(x_{i}\right)}-i^{-1 / \alpha\left(x_{i}\right)}\right) f\left(v, x_{i}, V_{i}\right), \\
Y_{i}^{2}=\gamma_{i} a\left(x_{i}\right)\left(\Gamma_{i}^{-1 / \alpha\left(x_{i}\right)}-i^{-1 / \alpha\left(x_{i}\right)}\right) f_{u}^{\prime}\left(v, x_{i}, V_{i}\right) \\
Y_{i}^{3}=\gamma_{i} a\left(x_{i}\right) \frac{\alpha^{\prime}\left(x_{i}\right)}{\alpha\left(x_{i}\right)^{2}}\left(\log \left(\Gamma_{i}\right) \Gamma_{i}^{-1 / \alpha\left(x_{i}\right)}-\log (i) i^{-1 / \alpha\left(x_{i}\right)}\right) f\left(v, x_{i}, V_{i}\right) .
\end{gathered}
$$

Note that each $w_{i}$ depends on $a, f, \alpha, u, v, V_{i}$, and each $x_{i}$ depends on $a, f, \alpha, u, v, V_{i}, \Gamma_{i}$ but not on $\gamma_{i}$. This remark will be useful in the sequel.

In [13], it is proved that each series $\sum_{i=1}^{\infty} Z_{i}^{j}$ and $\sum_{i=1}^{\infty} Y_{i}^{j}, j=1,2,3$, converges almost surely. Let $x>0$. We consider $\mathrm{P}\left(\left|\sum_{i=1}^{\infty} Z_{i}^{j}\right|>x\right)$ and $\mathrm{P}\left(\left|\sum_{i=1}^{\infty} Y_{i}^{j}\right|>x\right)$ for $j=1,2,3$.

Let $\eta \in\left(0, \min \left(\frac{2 c}{d}-1, \frac{c}{d}(\delta+1)-1\right)\right)$. Markov inequality yields

$$
\begin{aligned}
\mathrm{P}\left(\left|\sum_{i=1}^{\infty} Z_{i}^{j}\right|>x\right) & \leq \frac{1}{x^{d}} \mathrm{E}\left[\left|\sum_{i=1}^{\infty} Z_{i}^{j}\right|^{d}\right] \\
& \leq \frac{1}{x^{d}}\left(\mathrm{E}\left[\left|\sum_{i=1}^{\infty} Z_{i}^{j}\right|^{d(1+\eta)}\right]\right)^{\frac{1}{1+\eta}}
\end{aligned}
$$

The random variables $Z_{i}^{j}$ are independent with mean 0 thus, by Theorem 2 of [24]:

$$
\mathrm{E}\left[\left|\sum_{i=1}^{+\infty} Z_{i}^{j}\right|^{d(1+\eta)}\right] \leq 2 \sum_{i=1}^{+\infty} \mathrm{E}\left[\left|Z_{i}^{j}\right|^{d(1+\eta)}\right] .
$$

For $j=1$,

$$
\begin{aligned}
\mathrm{E}\left[\left|Z_{i}^{1}\right|^{d(1+\eta)}\right] & =\mathrm{E}\left[\left|a^{\prime}\left(w_{i}\right)\right|^{d(1+\eta)} i^{-\frac{d(1+\eta)}{\alpha\left(w_{i}\right)}}\left|f\left(v, w_{i}, V_{i}\right)\right|^{d(1+\eta)}\right] \\
& \leq \frac{K_{U}}{i^{1+\eta}} \mathrm{E}\left[\left(\sup _{w \in B(u, \varepsilon)}\left|f\left(v, w, V_{i}\right)\right|^{\alpha(w)}\right)^{\frac{d(1+\eta)}{\alpha\left(w_{i}\right)}}\right] \\
& \leq \frac{K_{U}}{i^{1+\eta}} \mathrm{E}\left[\left(\sup _{w \in B(u, \varepsilon)}\left|f\left(v, w, V_{1}\right)\right|^{\alpha(w)}\right)^{1+\eta}+\left(\sup _{w \in B(u, \varepsilon)}\left|f\left(v, w, V_{1}\right)\right|^{\alpha(w)}\right)^{\frac{d}{c}(1+\eta)}\right] \\
& \leq \frac{K_{U}}{i^{1+\eta}} .
\end{aligned}
$$


For $j=2$,

$$
\begin{aligned}
\mathrm{E}\left[\left|Z_{i}^{2}\right|^{d(1+\eta)}\right] & \leq \frac{K_{U}}{i^{1+\eta}} \mathrm{E}\left[\left(\sup _{w \in B(u, \varepsilon)}\left|f_{u}^{\prime}\left(v, w, V_{1}\right)\right|^{\alpha(w)}\right)^{1+\eta}+\left(\sup _{w \in B(u, \varepsilon)}\left|f_{u}^{\prime}\left(v, w, V_{1}\right)\right|^{\alpha(w)}\right)^{\frac{d}{c}(1+\eta)}\right] \\
& \leq \frac{K_{U}}{i^{1+\eta}}
\end{aligned}
$$

For $j=3$,

$$
\begin{aligned}
\mathrm{E}\left[\left|Z_{i}^{3}\right|^{d(1+\eta)}\right] & =\mathrm{E}\left[\left|a\left(w_{i}\right) \frac{\alpha^{\prime}\left(w_{i}\right)}{\alpha\left(w_{i}\right)^{2}}\right|^{d(1+\eta)}\left|f\left(v, w_{i}, V_{i}\right)\right|^{d(1+\eta)} \frac{(\log i)^{d(1+\eta)}}{i^{\frac{d(1+\eta)}{\alpha\left(w_{i}\right)}}}\right] \\
& \leq K_{U} \frac{(\log i)^{d(1+\eta)}}{i^{1+\eta}}
\end{aligned}
$$

Finally, $\sup _{v \in U} \sum_{i=1}^{+\infty} \mathrm{E}\left[\left|Z_{i}^{j}\right|^{d(1+\eta)}\right]<+\infty$ thus

$$
\mathrm{P}\left(\left|\sum_{i=1}^{\infty} Z_{i}^{j}\right|>x\right) \leq \frac{K_{U}}{x^{d}}
$$

We consider now $\mathrm{P}\left(\left|\sum_{i=1}^{\infty} Y_{i}^{j}\right|>x\right)$ for $j=1,2,3$.

$$
\mathrm{P}\left(\left|\sum_{i=1}^{\infty} Y_{i}^{j}\right|>x\right) \leq \mathrm{P}\left(\left|Y_{1}^{j}\right| \geq \frac{x}{2}\right)+\mathrm{P}\left(\left|\sum_{i=2}^{\infty} Y_{i}^{j}\right| \geq \frac{x}{2}\right)
$$

Since $\mathrm{P}\left(\left|\sum_{i=2}^{\infty} Y_{i}^{j}\right| \geq \frac{x}{2}\right) \leq \frac{2^{d}}{x^{d}}\left(\mathrm{E}\left[\left|\sum_{i=2}^{\infty} Y_{i}^{j}\right|^{d(1+\eta)}\right]\right)^{\frac{1}{1+\eta}}$, we want to apply Theorem 2 of [24] again. Let $S_{m}=\sum_{i=1}^{m} Y_{i}^{j}$ and write $Y_{i}^{j}=\gamma_{i} W_{i}^{j}$. Note that $\gamma_{i}$ is independent of $W_{i}^{j}$ and $S_{i-1}$.

$$
\begin{aligned}
\mathrm{E}\left(Y_{m+1}^{j} \mid S_{m}\right) & =\mathrm{E}\left(\mathrm{E}\left(Y_{m+1}^{j} \mid S_{m}, W_{m+1}\right) \mid S_{m}\right) \\
& =\mathrm{E}\left(\mathrm{E}\left(\gamma_{m+1} W_{m+1}^{j} \mid S_{m}, W_{m+1}\right) \mid S_{m}\right) \\
& =\mathrm{E}\left(W_{m+1}^{j} \mathrm{E}\left(\gamma_{m+1} \mid S_{m}, W_{m+1}\right) \mid S_{m}\right) \\
& =\mathrm{E}\left(W_{m+1}^{j} \mathrm{E}\left(\gamma_{m+1}\right) \mid S_{m}\right) \\
& =0 .
\end{aligned}
$$

We apply Theorem 2 of [24] with $(d(1+\eta)<2)$,

$$
\mathrm{E}\left[\left|\sum_{i=2}^{\infty} Y_{i}^{j}\right|^{d(1+\eta)}\right] \leq 2 \sum_{i=2}^{\infty} \mathrm{E}\left|Y_{i}^{j}\right|^{d(1+\eta)},
$$

and

$$
\mathrm{P}\left(\left|\sum_{i=1}^{\infty} Y_{i}^{j}\right|>x\right) \leq \mathrm{P}\left(\left|Y_{1}^{j}\right| \geq \frac{x}{2}\right)+\frac{2^{d}}{x^{d}}\left(2 \sum_{i=2}^{\infty} \mathrm{E}\left|Y_{i}^{j}\right|^{d(1+\eta)}\right)^{\frac{1}{1+\eta}} .
$$


For $j=1$,

$$
\begin{aligned}
\mathrm{P}\left(\left|Y_{1}^{1}\right| \geq \frac{x}{2}\right) & =\mathrm{P}\left(\left|a^{\prime}\left(x_{1}\right)\right|^{\alpha\left(x_{1}\right)}\left|\frac{1}{\Gamma_{1}^{1 / \alpha\left(x_{1}\right)}}-1\right|^{\alpha\left(x_{1}\right)}\left|f\left(v, x_{1}, V_{1}\right)\right|^{\alpha\left(x_{1}\right)} \geq \frac{x^{\alpha\left(x_{1}\right)}}{2^{\alpha\left(x_{1}\right)}}\right) \\
& \leq \mathrm{P}\left(\left|\frac{1}{\Gamma_{1}^{1 / \alpha\left(x_{1}\right)}}-1\right|^{\alpha\left(x_{1}\right)} \sup _{w \in B(u, \varepsilon)}\left|f\left(v, w, V_{1}\right)\right|^{\alpha(w)} \geq K_{U} x^{\alpha\left(x_{1}\right)}\right)
\end{aligned}
$$

For $x<1$,

$$
\begin{aligned}
\mathrm{P}\left(\left|Y_{1}^{1}\right| \geq \frac{x}{2}\right) \leq & \mathrm{P}\left(\left|\frac{1}{\Gamma_{1}^{1 / \alpha\left(x_{1}\right)}}-1\right|^{\alpha\left(x_{1}\right)} \sup _{w \in B(u, \varepsilon)}\left|f\left(v, w, V_{1}\right)\right|^{\alpha(w)} \geq K_{U} x^{d}\right) \\
\leq & \mathrm{P}\left(\left\{\sup _{w \in B(u, \varepsilon)}\left|f\left(v, w, V_{1}\right)\right|^{\alpha(w)} \geq K_{U} x^{d}\right\} \cap\left\{\Gamma_{1}>1\right\}\right) \\
& +\mathrm{P}\left(\left\{\left|\frac{1}{\Gamma_{1}}\right| \Gamma_{1}^{1 / \alpha\left(x_{1}\right)}-\left.1\right|^{\alpha\left(x_{1}\right)} \sup _{w \in B(u, \varepsilon)}\left|f\left(v, w, V_{1}\right)\right|^{\alpha(w)} \geq K_{U} x^{d}\right\} \cap\left\{\Gamma_{1} \leq 1\right\}\right) . \\
\mathrm{P}\left(\left\{\sup _{w \in B(u, \varepsilon)}\left|f\left(v, w, V_{1}\right)\right|^{\alpha(w)} \geq K_{U} x^{d}\right\} \cap\left\{\Gamma_{1}>1\right\}\right) \leq \frac{K_{U}}{x^{d}} \mathrm{E}\left(\sup _{w \in B(u, \varepsilon)}\left|f\left(v, w, V_{1}\right)\right|^{\alpha(w)}\right) & \leq \frac{K_{U}}{x^{d}} .
\end{aligned}
$$

Let $W(v, x)=\sup _{w \in B(u, \varepsilon)}|f(v, w, x)|^{\alpha(w)}$ and $F_{v, V_{1}}$ be the distribution of $W\left(v, V_{1}\right)$.

$$
\begin{aligned}
\mathrm{P}\left(\left\{\left|\frac{1}{\Gamma_{1}}\right| \Gamma_{1}^{1 / \alpha\left(x_{1}\right)}-\left.1\right|^{\alpha\left(x_{1}\right)} W\left(v, V_{1}\right) \geq K_{U} x^{d}\right\} \cap\left\{\Gamma_{1} \leq 1\right\}\right) & \leq \mathrm{P}\left(W\left(v, V_{1}\right) \geq K_{U} x^{d} \Gamma_{1}\right) \\
& =\int_{0}^{+\infty} \mathrm{P}\left(z \geq K_{U} x^{d} \Gamma_{1}\right) F_{v, V_{1}}(\mathrm{~d} z) \\
& =\int_{0}^{+\infty}\left(1-\mathrm{e}^{-\frac{z}{K_{U} x^{d}}}\right) F_{v, V_{1}}(\mathrm{~d} z) \\
& \leq \int_{0}^{+\infty} \frac{z}{K_{U} x^{d}} F_{v, V_{1}}(\mathrm{~d} z) \\
& \leq \frac{K_{U}}{x^{d}}
\end{aligned}
$$

For $x \geq 1$,

$$
\begin{aligned}
& \mathrm{P}\left(\left|Y_{1}^{1}\right| \geq \frac{x}{2}\right) \leq \mathrm{P}\left(\left|\frac{1}{\Gamma_{1}^{1 / \alpha\left(x_{1}\right)}}-1\right|^{\alpha\left(x_{1}\right)} \sup _{w \in B(u, \varepsilon)}\left|f\left(v, w, V_{1}\right)\right|^{\alpha(w)} \geq K_{U} x^{c}\right) \\
& \mathrm{P}\left(\left|Y_{1}^{1}\right| \geq \frac{x}{2}\right) \leq \frac{K_{U}}{x^{c}}
\end{aligned}
$$


For $i \geq 2$,

$$
\begin{aligned}
\mathrm{E}\left|Y_{i}^{1}\right|^{d(1+\eta)} & =\mathrm{E}\left(\left|a^{\prime}\left(x_{i}\right)\right|^{d(1+\eta)}\left|\Gamma_{i}^{-1 / \alpha\left(x_{i}\right)}-i^{-1 / \alpha\left(x_{i}\right)}\right|^{d(1+\eta)}\left(\left|f\left(v, x_{i}, V_{i}\right)\right|^{\alpha\left(x_{i}\right)}\right)^{\frac{d(1+\eta)}{\alpha\left(x_{i}\right)}}\right) \\
& \leq K_{U} \mathrm{E}\left(i^{-\frac{d(1+\eta)}{\alpha\left(x_{i}\right)}} W\left(v, V_{i}\right)^{\frac{d(1+\eta)}{\alpha\left(x_{i}\right)}}\left|\left(\frac{i}{\Gamma_{i}}\right)^{1 / \alpha\left(x_{i}\right)}-1\right|^{d(1+\eta)}\right) \\
& \leq \frac{K_{U}}{i^{1+\eta}} \mathrm{E}\left(\left[W\left(v, V_{i}\right)^{1+\eta}+W\left(v, V_{i}\right)^{\frac{d}{c}(1+\eta)}\right]\left[\left|\left(\frac{i}{\Gamma_{i}}\right)^{1 / c}-1\right|^{d(1+\eta)}+\left|\left(\frac{i}{\Gamma_{i}}\right)^{1 / d}-1\right|^{d(1+\eta)}\right]\right) \\
& \leq \frac{K_{U}}{i^{1+\eta}} \mathrm{E}\left(W\left(v, V_{i}\right)^{1+\eta}+W\left(v, V_{i}\right)^{\frac{d}{c}(1+\eta)}\right) \mathrm{E}\left(\left|\left(\frac{i}{\Gamma_{i}}\right)^{1 / c}-1\right|^{d(1+\eta)}+\left|\left(\frac{i}{\Gamma_{i}}\right)^{1 / d}-1\right|^{d(1+\eta)}\right) .
\end{aligned}
$$

Using the fact that $\eta \leq \delta$ and $\frac{d}{c}(1+\eta) \leq 1+\delta$,

$$
\begin{aligned}
\mathrm{E}\left(W\left(v, V_{i}\right)^{1+\eta}+W\left(v, V_{i}\right)^{\frac{d}{c}(1+\eta)}\right) & =\mathrm{E}\left(W\left(v, V_{1}\right)^{1+\eta}+W\left(v, V_{1}\right)^{\frac{d}{c}(1+\eta)}\right) \\
& \leq K_{U} \\
\mathrm{E}\left|\left(\frac{i}{\Gamma_{i}}\right)^{1 / c}-1\right|^{d(1+\eta)} & \leq K_{U}\left(1+\mathrm{E}\left(\left(\frac{i}{\Gamma_{i}}\right)^{\frac{d}{c}(1+\eta)}\right)\right) \\
& \leq K_{U},
\end{aligned}
$$

and

$$
\mathrm{E}\left|\left(\frac{i}{\Gamma_{i}}\right)^{1 / d}-1\right|^{d(1+\eta)} \leq K_{U}
$$

As a consequence:

$$
\sup _{v \in U} \sum_{i=2}^{+\infty} \mathrm{E}\left|Y_{i}^{1}\right|^{d(1+\eta)} \leq K_{U}
$$

and

$$
\mathrm{P}\left(\left|\sum_{i=1}^{\infty} Y_{i}^{1}\right|>x\right) \leq K_{U}\left(\frac{1}{x^{c}}+\frac{1}{x^{d}}\right) .
$$

For $j=2$, since the conditions required on $\left(a^{\prime}, f\right)$ are also satisfied by $\left(a, f^{\prime} u\right)$, one gets in a similar fashion

$$
\mathrm{P}\left(\left|\sum_{i=1}^{\infty} Y_{i}^{2}\right|>x\right) \leq K_{U}\left(\frac{1}{x^{c}}+\frac{1}{x^{d}}\right) .
$$

For $j=3$,

$$
\begin{aligned}
\mathrm{P}\left(\left|Y_{1}^{3}\right| \geq \frac{x}{2}\right) & =\mathrm{P}\left(\left|a\left(x_{1}\right) \frac{\alpha^{\prime}\left(x_{1}\right)}{\alpha\left(x_{1}\right)^{2}} \log \left(\Gamma_{1}\right) \Gamma_{1}^{-1 / \alpha\left(x_{1}\right)} f\left(v, x_{1}, V_{1}\right)\right| \geq \frac{x}{2}\right) \\
& \leq \mathrm{P}\left(K_{U} \frac{\left|f\left(v, x_{1}, V_{1}\right)\right|^{\alpha\left(x_{1}\right)}}{x^{\alpha\left(x_{1}\right)}} \geq \frac{\Gamma_{1}}{\left|\log \Gamma_{1}\right|^{\alpha\left(x_{1}\right)}}\right) .
\end{aligned}
$$

Let $g(z)=\frac{z}{|\log z|^{\alpha\left(x_{1}\right)}}$, for $z<1$. 
$g$ is a one-to-one increasing function, and for all $z<1$ such that $z|\log z|^{\alpha\left(x_{1}\right)}<1$ and $\mid 1+$ $\left.\alpha\left(x_{1}\right) \frac{\log |\log z|}{|\log z|}\right|^{\alpha\left(x_{1}\right)} \leq 2$,

$$
g\left(z|\log z|^{\alpha\left(x_{1}\right)}\right)=\frac{z|\log z|^{\alpha\left(x_{1}\right)}}{\left|\log z+\alpha\left(x_{1}\right) \log \right| \log z||^{\alpha\left(x_{1}\right)}} \geq \frac{z}{2}
$$

thus $g^{-1}\left(\frac{z}{2}\right) \leq z|\log z|^{\alpha\left(x_{1}\right)}$.

Fix $A>0$ such that for all $0<z<A, g^{-1}(z) \leq 2 z|\log 2+\log z|^{\alpha\left(x_{1}\right)}$ i.e.

$$
g^{-1}(z) \leq K_{U} z|\log z|^{\alpha\left(x_{1}\right)} .
$$

Let $B=\left\{K_{U} \frac{\left|f\left(v, x_{1}, V_{1}\right)\right|^{\alpha\left(x_{1}\right)}}{x^{\alpha\left(x_{1}\right)}} \geq \frac{\Gamma_{1}}{\left|\log \Gamma_{1}\right|^{\alpha\left(x_{1}\right)}}\right\}$.

$$
\begin{aligned}
\mathrm{P}(B)= & \mathrm{P}\left(B \cap\left\{\Gamma_{1}>1\right\}\right)+\mathrm{P}\left(B \cap\left\{\Gamma_{1}<1\right\} \cap\left\{0 \leq K_{U} \frac{\left|f\left(v, x_{1}, V_{1}\right)\right|^{\alpha\left(x_{1}\right)}}{x^{\alpha\left(x_{1}\right)}} \leq A\right\}\right) \\
& +\mathrm{P}\left(B \cap\left\{\Gamma_{1}<1\right\} \cap\left\{K_{U} \frac{\left|f\left(v, x_{1}, V_{1}\right)\right|^{\alpha\left(x_{1}\right)}}{x^{\alpha\left(x_{1}\right)}}>A\right\}\right) .
\end{aligned}
$$

Each of these three terms will be treated separately.

$$
\begin{aligned}
-\mathrm{P}\left(B \cap\left\{\Gamma_{1}>1\right\}\right) & \leq \mathrm{P}\left(K_{U} \frac{\left|f\left(v, x_{1}, V_{1}\right)\right|^{\alpha\left(x_{1}\right)}}{x^{\alpha\left(x_{1}\right)}}\left|\log \Gamma_{1}\right|^{\alpha\left(x_{1}\right)} \geq 1\right) \\
& \leq \mathrm{P}\left(K_{U} \sup _{w \in B(u, \varepsilon)}\left|f\left(v, w, V_{1}\right)\right|^{\alpha(w)}\left(\left|\log \Gamma_{1}\right|^{c}+\left|\log \Gamma_{1}\right|^{d}\right) \geq x^{\alpha\left(x_{1}\right)}\right) .
\end{aligned}
$$

For $x \geq 1$,

$$
\begin{aligned}
\mathrm{P}\left(B \cap\left\{\Gamma_{1}>1\right\}\right) & \leq \mathrm{P}\left(K_{U} \sup _{w \in B(u, \varepsilon)}\left|f\left(v, w, V_{1}\right)\right|^{\alpha(w)}\left(\left|\log \Gamma_{1}\right|^{c}+\left|\log \Gamma_{1}\right|^{d}\right) \geq x^{c}\right) \\
& \leq \frac{K_{U}}{x^{c}} \mathrm{E}\left(\sup _{w \in B(u, \varepsilon)}\left|f\left(v, w, V_{1}\right)\right|^{\alpha(w)}\right) \mathrm{E}\left(\left|\log \Gamma_{1}\right|^{c}+\left|\log \Gamma_{1}\right|^{d}\right) \\
& \leq \frac{K_{U}}{x^{c}} .
\end{aligned}
$$

For $x<1$,

$$
\begin{gathered}
\mathrm{P}\left(B \cap\left\{\Gamma_{1}>1\right\}\right) \leq \mathrm{P}\left(K_{U} \sup _{w \in B(u, \varepsilon)}\left|f\left(v, w, V_{1}\right)\right|^{\alpha(w)}\left(\left|\log \Gamma_{1}\right|^{c}+\left|\log \Gamma_{1}\right|^{d}\right) \geq x^{d}\right) \\
\leq \frac{K_{U}}{x^{d}} . \\
\text { - } \mathrm{P}\left(B \cap\left\{\Gamma_{1}<1\right\} \cap\left\{K_{U} \frac{\left|f\left(v, x_{1}, V_{1}\right)\right|^{\alpha\left(x_{1}\right)}}{x^{\alpha\left(x_{1}\right)}}>A\right\}\right) \leq \mathrm{P}\left(K_{U}\left|f\left(v, x_{1}, V_{1}\right)\right|^{\alpha\left(x_{1}\right)} \geq A x^{\alpha\left(x_{1}\right)}\right) \\
\leq \frac{K_{U}}{x^{c}}+\frac{K_{U}}{x^{d}} .
\end{gathered}
$$




$$
\begin{aligned}
-\mathrm{P}\left(B \cap\left\{\Gamma_{1}<1\right\}\right. & \left.\cap\left\{0 \leq K_{U} \frac{\left|f\left(v, x_{1}, V_{1}\right)\right|^{\alpha\left(x_{1}\right)}}{x^{\alpha\left(x_{1}\right)}} \leq A\right\}\right) \\
& =\mathrm{P}\left(\left\{g\left(\Gamma_{1}\right) \leq K_{U} \frac{\left|f\left(v, x_{1}, V_{1}\right)\right|^{\alpha\left(x_{1}\right)}}{x^{\alpha\left(x_{1}\right)}}\right\} \cap\left\{\Gamma_{1}<1\right\} \cap\left\{0 \leq K_{U} \frac{\left|f\left(v, x_{1}, V_{1}\right)\right|^{\alpha\left(x_{1}\right)}}{x^{\alpha\left(x_{1}\right)}} \leq A\right\}\right) \\
& \leq \mathrm{P}\left(\Gamma_{1} \leq K_{U} \frac{\left|f\left(v, x_{1}, V_{1}\right)\right|^{\alpha\left(x_{1}\right)}}{x^{\alpha\left(x_{1}\right)}}+K_{U} \frac{\left|f\left(v, x_{1}, V_{1}\right)\right|^{\alpha\left(x_{1}\right)}}{x^{\alpha\left(x_{1}\right)}}\left|\log \frac{\left|f\left(v, x_{1}, V_{1}\right)\right|^{\alpha\left(x_{1}\right)}}{x^{\alpha\left(x_{1}\right)}}\right|^{\alpha\left(x_{1}\right)}\right) \\
& \leq \mathrm{P}\left(\Gamma_{1} \leq K_{U}\left|f\left(v, x_{1}, V_{1}\right)\right|^{\alpha\left(x_{1}\right)}\left(\frac{1+|\log x|^{\alpha\left(x_{1}\right)}}{x^{\alpha\left(x_{1}\right)}}\right)+K_{U} \frac{|| f\left(v, x_{1}, V_{1}\right)|\log | f\left(v, x_{1}, V_{1}\right)||^{\alpha\left(x_{1}\right)}}{x^{\alpha\left(x_{1}\right)}}\right) .
\end{aligned}
$$

With $W(v, x)=\sup _{w \in B(u, \varepsilon)}|f(v, w, x)|^{\alpha(w)}$ and $Z(v, x)=\left.\sup _{w \in B(u, \varepsilon)}|f(v, w, x) \log | f(v, w, x)\right|^{\alpha(w)}$,

$$
\begin{aligned}
\mathrm{P}\left(B \cap\left\{\Gamma_{1}<1\right\} \cap\right. & \left.\left\{0 \leq K_{U} \frac{\left|f\left(v, x_{1}, V_{1}\right)\right|^{\alpha\left(x_{1}\right)}}{x^{\alpha\left(x_{1}\right)}} \leq A\right\}\right) \\
& \leq \mathrm{P}\left(\Gamma_{1} \leq K_{U} W\left(v, V_{1}\right)\left(\frac{1+|\log x|^{\alpha\left(x_{1}\right)}}{x^{\alpha\left(x_{1}\right)}}\right)+K_{U} \frac{Z\left(v, V_{1}\right)}{x^{\alpha\left(x_{1}\right)}}\right) \\
& \leq \mathrm{P}\left(\Gamma_{1} \leq K_{U} W\left(v, V_{1}\right)\left(\frac{1+|\log x|^{\alpha\left(x_{1}\right)}}{x^{\alpha\left(x_{1}\right)}}\right)\right)+\mathrm{P}\left(\Gamma_{1} \leq K_{U} Z\left(v, V_{1}\right)\left(\frac{1+|\log x|^{\alpha\left(x_{1}\right)}}{x^{\alpha\left(x_{1}\right)}}\right)\right)
\end{aligned}
$$

Since $\frac{1+|\log x|^{\alpha\left(x_{1}\right)}}{x^{\alpha\left(x_{1}\right)}} \leq K_{U}\left(\frac{1+|\log x|^{c}}{x^{c}}+\frac{1+|\log x|^{d}}{x^{d}}\right)$

$$
\begin{aligned}
\mathrm{P}\left(\Gamma_{1} \leq K_{U} W\left(v, V_{1}\right)\left(\frac{1+|\log x|^{\alpha\left(x_{1}\right)}}{x^{\alpha\left(x_{1}\right)}}\right)\right) & \leq \mathrm{P}\left(\Gamma_{1} \leq K_{U} W\left(v, V_{1}\right)\left(\frac{1+|\log x|^{c}}{x^{c}}+\frac{1+|\log x|^{d}}{x^{d}}\right)\right) \\
& \leq K_{U}\left(\frac{1+|\log x|^{c}}{x^{c}}+\frac{1+|\log x|^{d}}{x^{d}}\right),
\end{aligned}
$$

and

$$
\mathrm{P}\left(\Gamma_{1} \leq K_{U} Z\left(v, V_{1}\right)\left(\frac{1+|\log x|^{\alpha\left(x_{1}\right)}}{x^{\alpha\left(x_{1}\right)}}\right)\right) \leq \mathrm{P}\left(\Gamma_{1} \leq K_{U} Z\left(v, V_{1}\right)\left(\frac{1+|\log x|^{c}}{x^{c}}+\frac{1+|\log x|^{d}}{x^{d}}\right)\right)
$$


Denoting $G_{v, V_{1}}$ the distribution of $Z\left(v, V_{1}\right)$,

$$
\begin{aligned}
\mathrm{P}\left(\Gamma_{1} \leq K_{U} Z\left(v, V_{1}\right)\right. & \left.\left(\frac{1+|\log x|^{c}}{x^{c}}+\frac{1+|\log x|^{d}}{x^{d}}\right)\right) \\
& =\int_{0}^{+\infty}\left(1-\exp \left(-K_{U}\left(\frac{1+|\log x|^{c}}{x^{c}}+\frac{1+|\log x|^{d}}{x^{d}}\right) z\right)\right) G_{v, V_{1}}(\mathrm{~d} z) \\
& \leq K_{U}\left(\frac{1+|\log x|^{c}}{x^{c}}+\frac{1+|\log x|^{d}}{x^{d}}\right) \int_{0}^{+\infty} z G_{v, V_{1}}(\mathrm{~d} z) \\
& \leq K_{U}\left(\frac{1+|\log x|^{c}}{x^{c}}+\frac{1+|\log x|^{d}}{x^{d}}\right)
\end{aligned}
$$

since $\sup _{v \in B(u, \varepsilon)} \mathrm{E}\left(Z\left(v, V_{1}\right)\right)<+\infty$.

Finally,

$$
\mathrm{P}\left(B \cap\left\{\Gamma_{1}<1\right\} \cap\left\{0 \leq K_{U} \frac{\left|f\left(v, x_{1}, V_{1}\right)\right|^{\alpha\left(x_{1}\right)}}{x^{\alpha\left(x_{1}\right)}} \leq A\right\}\right) \leq K_{U}\left(\frac{1+|\log x|^{c}}{x^{c}}+\frac{1+|\log x|^{d}}{x^{d}}\right)
$$

and

$$
\mathrm{P}\left(\left|Y_{1}^{3}\right| \geq \frac{x}{2}\right) \leq K_{U}\left(\frac{1+|\log x|^{c}}{x^{c}}+\frac{1+|\log x|^{d}}{x^{d}}\right) .
$$

For $i \geq 2$,

$$
\begin{aligned}
\mathrm{E}\left|Y_{i}^{3}\right|^{d(1+\eta)} & \leq K_{U} \frac{|\log i|^{d(1+\eta)}}{i^{1+\eta}} \mathrm{E}\left(W\left(v, V_{i}\right)^{1+\eta}+W\left(v, V_{i}\right)^{\frac{d}{c}(1+\eta)}\right) \mathrm{E}\left(\left|\frac{\log \Gamma_{i}}{\log i}\right|\left|\left(\frac{i}{\Gamma_{i}}\right)^{1 / \alpha\left(x_{i}\right)}-1\right|^{d(1+\eta)}\right) \\
& \leq K_{U} \frac{|\log i|^{d(1+\eta)}}{i^{1+\eta}} \mathrm{E}\left(\left|\frac{\log \Gamma_{i}}{\log i}\right|\left|\left(\frac{i}{\Gamma_{i}}\right)^{1 / \alpha\left(x_{i}\right)}-1\right|^{d(1+\eta)}\right) \\
& \leq K_{U} \frac{|\log i|^{d(1+\eta)}}{i^{1+\eta}} \mathrm{E}\left(\left|\frac{\log \Gamma_{i}}{\log i}\right|\left|\left(\frac{i}{\Gamma_{i}}\right)^{1 / c}-1\right|^{d(1+\eta)}+\left|\frac{\log \Gamma_{i}}{\log i}\right|\left|\left(\frac{i}{\Gamma_{i}}\right)^{1 / d}-1\right|^{d(1+\eta)}\right) \\
& \leq K_{U} \frac{|\log i|^{d(1+\eta)}}{i^{1+\eta}},
\end{aligned}
$$

thus

$$
\sup _{v \in U} \sum_{i=2}^{+\infty} \mathrm{E}\left|Y_{i}^{3}\right|^{d(1+\eta)} \leq K_{U}
$$

and

$$
\mathrm{P}\left(\left|\sum_{i=1}^{\infty} Y_{i}^{3}\right|>x\right) \leq K_{U}\left(\frac{1+|\log x|^{c}}{x^{c}}+\frac{1+|\log x|^{d}}{x^{d}}\right)
$$


Let us go back to $\mathrm{P}(|X(v, v)-X(v, u)|>x)$.

$$
\begin{aligned}
\mathrm{P}(|X(v, v)-X(v, u)|>x) & =\mathrm{P}\left(|u-v|\left|\sum_{i=1}^{\infty}\left(Z_{i}^{1}+Z_{i}^{2}+Z_{i}^{3}+Y_{i}^{1}+Y_{i}^{2}+Y_{i}^{3}\right)\right|>x\right) \\
& \leq \sum_{j=1}^{3}\left(\mathrm{P}\left(\left|\sum_{i=1}^{\infty} Z_{i}^{j}\right| \geq \frac{x}{6|u-v|}\right)+\mathrm{P}\left(\left|\sum_{i=1}^{\infty} Y_{i}^{j}\right| \geq \frac{x}{6|u-v|}\right)\right) \\
& \leq K_{U}\left(\frac{|v-u|^{d}}{x^{d}}\left(1+\left|\log \frac{|v-u|}{x}\right|^{d}\right)+\frac{|v-u|^{c}}{x^{c}}\left(1+\left|\log \frac{|v-u|}{x}\right|\right)\right)
\end{aligned}
$$

and the proof is complete.

General case: we apply the previous result to the function $g(t, w, x)=r(x)^{1 / \alpha(w)} f(t, w, x)$ on $(E, \mathcal{E}, \hat{m})$ where $\hat{m}(\mathrm{~d} x)=\frac{1}{r(x)} m(\mathrm{~d} x)$. We check that $g$ is satisfying the conditions (R1), (M1), and (M2) with $r(x) \equiv 1$.

- By (R1), the family of functions $v \rightarrow f(t, v, x)$ is differentiable for all $(v, t)$ in $U^{2}$ and almost all $x$ in $E$ thus $v \rightarrow g(t, v, x)$ is differentiable too i.e. (R1) holds for $g$.

- Choose $\delta>\frac{d}{c}-1$ such that (M1) holds.

$$
\sup _{w \in U}\left(|g(t, w, x)|^{\alpha(w)}\right)=r(x) \sup _{w \in U}\left(|f(t, w, x)|^{\alpha(w)}\right) .
$$

One has

$$
\begin{aligned}
\int_{\mathbf{R}}\left[\sup _{w \in U}\left(|g(t, w, x)|^{\alpha(w)}\right)\right]^{1+\delta} \hat{m}(\mathrm{~d} x) & =\int_{\mathbf{R}} r(x)^{1+\delta}\left[\sup _{w \in U}\left(|f(t, w, x)|^{\alpha(w)}\right)\right]^{1+\delta} \hat{m}(\mathrm{~d} x) \\
& =\int_{\mathbf{R}}\left[\sup _{w \in U}\left(|f(t, w, x)|^{\alpha(w)}\right)\right]^{1+\delta} r(x)^{\delta} m(\mathrm{~d} x)
\end{aligned}
$$

thus (M1) holds for $g$.

- Choose $\delta>\frac{d}{c}-1$ such that (M2) and (M3) hold.

$$
g_{u}^{\prime}(t, w, x)=r(x)^{1 / \alpha(w)}\left(f_{u}^{\prime}(t, w, x)-\frac{\alpha^{\prime}(w)}{\alpha^{2}(w)} \log (r(x)) f(t, w, x)\right)
$$

and

$$
\begin{aligned}
\int_{\mathbf{R}}\left[\sup _{w \in U}\left(\left|g_{u}^{\prime}(t, w, x)\right|^{\alpha(w)}\right)\right]^{1+\delta} & \hat{m}(\mathrm{~d} x) \\
& \leq \int_{\mathbf{R}}\left[\sup _{w \in U}\left[\left|f_{u}^{\prime}(t, w, x)-\frac{\alpha^{\prime}(w)}{\alpha^{2}(w)} \log (r(x)) f(t, w, x)\right|^{\alpha(w)}\right]\right]^{1+\delta} r(x)^{\delta} m(\mathrm{~d} x) .
\end{aligned}
$$

The inequality $|a+b|^{\delta} \leq \max \left(1,2^{\delta-1}\right)\left(|a|^{\delta}+|b|^{\delta}\right)$ shows that (M2) holds for $g$.

\subsection{Proof of Theorem 3.1}

Consider

$$
\mathrm{E}\left[\left|\frac{Y(t+\varepsilon)-Y(t)}{\varepsilon^{h(t)}}\right|^{\eta}\right]=\int_{0}^{\infty} \mathrm{P}\left(\left|\frac{Y(t+\varepsilon)-Y(t)}{\varepsilon^{h(t)}}\right|^{\eta}>x\right) \mathrm{d} x
$$


Thanks to (R1), (M1), (M2) and (M3), $Y$ is $h(t)$-localisable at $t$ [13], thus for all $x>0$,

$$
\mathrm{P}\left(\left|\frac{Y(t+\varepsilon)-Y(t)}{\varepsilon^{h(t)}}\right|^{\eta}>x\right) \rightarrow \mathrm{P}\left(\left|Y_{t}^{\prime}(1)\right|^{\eta}>x\right) .
$$

We shall make use of Lebesgue dominated convergence theorem.

For $x \leq 1, \mathrm{P}\left(\left|\frac{Y(t+\varepsilon)-Y(t)}{\varepsilon^{h(t)}}\right|^{\eta}>x\right) \leq 1$.

For $x>1$,

$$
\begin{aligned}
\mathrm{P}\left(\left|\frac{Y(t+\varepsilon)-Y(t)}{\varepsilon^{h(t)}}\right|^{\eta}>x\right)= & \mathrm{P}\left(\left|\frac{Y(t+\varepsilon)-Y(t)}{\varepsilon^{h(t)}}\right|>x^{1 / \eta}\right) \\
\leq & \mathrm{P}\left(\left|\frac{X(t+\varepsilon, t+\varepsilon)-X(t+\varepsilon, t)}{\varepsilon^{h(t)}}\right|>\frac{x^{1 / \eta}}{2}\right) \\
& +\mathrm{P}\left(\left|\frac{X(t+\varepsilon, t)-X(t, t)}{\varepsilon^{h(t)}}\right|>\frac{x^{1 / \eta}}{2}\right) .
\end{aligned}
$$

For the first term, by Proposition 4.11,

$$
\mathrm{P}\left(\left|\frac{X(t+\varepsilon, t+\varepsilon)-X(t+\varepsilon, t)}{\varepsilon^{h(t)}}\right|>\frac{x^{1 / \eta}}{2}\right) \leq \frac{K_{U}}{x^{d / \eta}}\left(1+|\log x|^{d}\right)+\frac{K_{U}}{x^{c / \eta}}\left(1+|\log x|^{c}\right) .
$$

For the second term, let $p \in(\eta, \alpha(t))$.

$$
\mathrm{P}\left(\left|\frac{X(t+\varepsilon, t)-X(t, t)}{\varepsilon^{h(t)}}\right|>\frac{x^{1 / \eta}}{2}\right)=\mathrm{P}\left(\left|\frac{X(t+\varepsilon, t)-X(t, t)}{\varepsilon^{h(t)}}\right|^{p}>\frac{x^{p / \eta}}{2^{p}}\right) .
$$

With Markov inequality and (H2),

$$
\begin{aligned}
\mathrm{P}\left(\left|\frac{X(t+\varepsilon, t)-X(t, t)}{\varepsilon^{h(t)}}\right|>\frac{x^{1 / \eta}}{2}\right) & \leq \frac{2^{p}}{x^{p / \eta} \varepsilon^{p h(t)}} C_{\alpha(t), 0}(p)^{p}\|f(t+\varepsilon, t, .)-f(t, t, .)\|_{\alpha(t)}^{p} \\
& \leq \frac{2^{p} C_{\alpha(t), 0}(p)^{p}}{x^{p / \eta} \varepsilon^{p h(t)}}\left(\int_{\mathbf{R}}|f(t+\varepsilon, t, x)-f(t, t, x)|^{\alpha(t)} m(\mathrm{~d} x)\right)^{p / \alpha(t)} \\
& \leq \frac{K_{p, \alpha(t)}}{x^{p / \eta}}
\end{aligned}
$$

thus

$$
\mathrm{P}\left(\left|\frac{Y(t+\varepsilon)-Y(t)}{\varepsilon^{h}}\right|^{\eta}>x\right) \leq K_{U}\left(\frac{1}{x^{d / \eta}}\left(1+|\log x|^{d}\right)+\frac{1}{x^{c / \eta}}\left(1+|\log x|^{c}\right)+\frac{1}{x^{p / \eta}}\right) \mathbf{1}_{x>1}+\mathbf{1}_{x \leq 1} .
$$

\subsection{Proof of Theorem 3.2}

To prove Theorem 3.2, we need a series of lemmas. More precisely, the proof is organized as follows: Lemmas 5.12 and 5.13 below are used to prove Lemma 5.15. Lemmas 5.14 and 5.15 allow to show Proposition 4.10. Finally, Propositions 4.10 and 4.9 are used to prove Theorem 3.2. 
Lemma 5.12. Assume (M6), (H4) and (H5). There exists a function $l \geq 0$ such that

$$
\lim _{r \rightarrow 0} \sup _{t \in U}|\Delta(r, t)-l(t)|=0
$$

where

$$
\Delta(r, t)=: \frac{1}{r^{2 h(t)}} \int_{\mathbf{R}} \int_{\frac{K}{r}}\left|\frac{C_{\alpha(t+r)}^{1 / \alpha(t+r)}}{y^{1 / \alpha(t+r)}} f(t+r, t+r, x)-\frac{C_{\alpha(t)}^{1 / \alpha(t)}}{y^{1 / \alpha(t)}} f(t, t, x)\right|^{2} \mathrm{~d} y m(\mathrm{~d} x) .
$$

Proof. Let $l(t)=\frac{C_{\alpha(t)}^{2 / \alpha(t)} K^{1-\frac{2}{\alpha(t)}}}{\frac{2}{\alpha(t)}-1} g(t)$. Note that condition (H4) implies the following:

$$
\exists K_{U}>0, \forall v \in U, \forall u \in U, \frac{1}{|v-u|^{1+2\left(h(u)-\frac{1}{\alpha(u)}\right)}} \int_{\mathbf{R}}|f(v, u, x)-f(u, u, x)|^{2} m(\mathrm{~d} x) \leq K_{U} .
$$

Expanding the square, we can write $\Delta(r, t)-l(t)=\Delta_{1}(r, t)+\Delta_{2}(r, t)+\Delta_{3}(r, t)$ where

$$
\begin{gathered}
\Delta_{1}(r, t)=\frac{1}{r^{2 h(t)}} \int_{\mathbf{R}} \int_{\frac{K}{r}}\left|\frac{C_{\alpha(t+r)}^{1 / \alpha(t+r)}}{y^{1 / \alpha(t+r)}} f(t+r, t+r, x)-\frac{C_{\alpha(t)}^{1 / \alpha(t)}}{y^{1 / \alpha(t)}} f(t+r, t, x)\right|^{2} \mathrm{~d} y m(\mathrm{~d} x), \\
\Delta_{2}(r, t)=\frac{2 C_{\alpha(t)}^{1 / \alpha(t)}}{r^{2 h(t)}} \int_{\mathbf{R}} \int_{\frac{K}{r}} \frac{1}{y^{1 / \alpha(t)}} g_{1}(r, t, x, y) g_{2}(r, t, x) \mathrm{d} y m(\mathrm{~d} x)
\end{gathered}
$$

and

$$
\Delta_{3}(r, t)=\frac{1}{r^{2 h(t)}} \int_{\mathbf{R}} \int_{\frac{K}{r}} \frac{C_{\alpha(t)}^{2 / \alpha(t)}}{y^{2 / \alpha(t)}}(f(t+r, t, x)-f(t, t, x))^{2} \mathrm{~d} y m(\mathrm{~d} x)-l(t),
$$

with $g_{1}(r, t, x, y)=\frac{C_{\alpha(t+r)}^{1 / \alpha(t+r)}}{y^{1 / \alpha(t+r)}} f(t+r, t+r, x)-\frac{C_{\alpha(t)}^{1 / \alpha(t)}}{y^{1 / \alpha(t)}} f(t+r, t, x)$ and $g_{2}(r, t, x)=f(t+r, t, x)-f(t, t, x)$. Since $\alpha$ is continuous, there exists a positive constant $K_{U}$ (that may change from line to line) such that

$$
\begin{aligned}
& \left|\Delta_{2}(r, t)\right| \leq \frac{K_{U}}{r^{2 h(t)}} \int_{\mathbf{R}} \int_{\frac{K}{r}}\left|\frac{g_{1}(r, t, x, y) g_{2}(r, t, x)}{y^{1 / \alpha(t)}}\right| \mathrm{d} y m(\mathrm{~d} x) \\
& \leq \frac{K_{U}}{r^{2 h(t)}}\left(\int_{\mathbf{R}} \int_{\frac{K}{r}}\left|g_{1}(r, t, x, y)\right|^{2} \mathrm{~d} y m(\mathrm{~d} x)\right)^{\frac{1}{2}}\left(\int_{\mathbf{R}} \int_{\frac{K}{r}}\left|\frac{g_{2}(r, t, x)}{y^{1 / \alpha(t)}}\right|^{2} \mathrm{~d} y m(\mathrm{~d} x)\right)^{\frac{1}{2}} \\
& \leq \frac{K_{U}}{r^{2 h(t)}} r^{h(t)} \sqrt{\Delta_{1}(r, t)}\left(\int_{\mathbf{R}} \int_{\frac{K}{r}}\left|\frac{g_{2}(r, t, x)}{y^{1 / \alpha(t)}}\right|^{2} \mathrm{~d} y m(\mathrm{~d} x)\right)^{\frac{1}{2}} \\
& \leq \frac{K_{U}}{r^{h(t)}} \sqrt{\Delta_{1}(r, t)}\left(\int_{\mathbf{R}}\left|g_{2}(r, t, x)\right|^{2} m(\mathrm{~d} x)\right)^{\frac{1}{2}} r^{\frac{1}{\alpha(t)}-\frac{1}{2}} K^{\frac{1}{2}-\frac{1}{\alpha(t)}} \sqrt{\frac{\alpha(t)}{2-\alpha(t)}} \\
& \leq K_{U} \sqrt{\Delta_{1}(r, t)}\left(\frac{1}{r^{1+2\left(h(t)-\frac{1}{\alpha(t)}\right)}} \int_{\mathbf{R}}|f(t+r, t, x)-f(t, t, x)|^{2} m(\mathrm{~d} x)\right)^{\frac{1}{2}} \\
& \leq K_{U} \sqrt{\Delta_{1}(r, t)} \text { with (5.11). }
\end{aligned}
$$


Let us show that $\lim _{r \rightarrow 0} \sup _{t \in U} \sqrt{\Delta_{1}(r, t)}=0$. The triangle inequality yields $\left.\sqrt{\Delta_{1}(r, t)}\right) \leq \delta_{1}(r, t)+\delta_{2}(r, t)+$ $\delta_{3}(r, t)$ where

$$
\begin{aligned}
& \delta_{1}(r, t)=\frac{1}{2 r^{h(t)}}\left(\int_{\mathbf{R}} \int_{\frac{K}{r}}\left|C_{\alpha(t+r)}^{1 / \alpha(t+r)}-C_{\alpha(t)}^{1 / \alpha(t)}\right|^{2} \frac{|f(t+r, t+r, x)|^{2}}{y^{2 / \alpha(t+r)}} \mathrm{d} y m(\mathrm{~d} x)\right)^{\frac{1}{2}}, \\
& \delta_{2}(r, t)=\frac{1}{2 r^{h(t)}}\left(\int_{\mathbf{R}} \int_{\frac{K}{r}} \frac{C_{\alpha(t)}^{2 / \alpha(t)}}{y^{2 / \alpha(t+r)}}|f(t+r, t+r, x)-f(t+r, t, x)|^{2} \mathrm{~d} y m(\mathrm{~d} x)\right)^{\frac{1}{2}},
\end{aligned}
$$

and

$$
\delta_{3}(r, t)=\frac{1}{2 r^{h(t)}}\left(\int_{\mathbf{R}} \int_{\frac{K}{r}} C_{\alpha(t)}^{2 / \alpha(t)}|f(t+r, t, x)|^{2}\left(\frac{1}{y^{1 / \alpha(t+r)}}-\frac{1}{y^{1 / \alpha(t)}}\right)^{2} \mathrm{~d} y m(\mathrm{~d} x)\right)^{\frac{1}{2}} .
$$

Now,

$$
\delta_{1}(r, t) \leq K_{U} \frac{\left|C_{\alpha(t+r)}^{1 / \alpha(t+r)}-C_{\alpha(t)}^{1 / \alpha(t)}\right|}{r^{h(t)}}\left(\frac{1}{\frac{2}{\alpha(t+r)}-1}\left(\frac{K}{r}\right)^{1-\frac{2}{\alpha(t+r)}}\right)^{\frac{1}{2}}\left(\int_{\mathbf{R}}|f(t+r, t+r, x)|^{2} m(\mathrm{~d} x)\right)^{\frac{1}{2}} .
$$

Since the function $u \mapsto C_{\alpha(u)}^{1 / \alpha(u)}$ is a $C^{1}$ function,

$$
\begin{aligned}
\delta_{1}(r, t) & \leq K_{U} r^{1-h(t)+\frac{1}{\alpha(t+r)}-\frac{1}{2}}\left(\int_{\mathbf{R}}|f(t+r, t+r, x)|^{2} m(\mathrm{~d} x)\right)^{\frac{1}{2}} \\
& \leq K_{U} r^{1-h(t)+\frac{1}{\alpha(t+r)}-\frac{1}{2}} \text { with }(\mathrm{M} 6) \\
& \leq K_{U} r^{\frac{1}{2}+\frac{1}{d}-h_{+}} .
\end{aligned}
$$

Since $\frac{1}{2}+\frac{1}{d}-h_{+}>0, \lim _{r \rightarrow 0} \sup _{t \in U} \delta_{1}(r, t)=0$.

$$
\begin{aligned}
\delta_{2}(r, t) & \leq \frac{C_{\alpha(t)}^{1 / \alpha(t)}}{2 r^{h(t)}}\left(\frac{1}{\frac{2}{\alpha(t+r)}-1}\left(\frac{K}{r}\right)^{1-\frac{2}{\alpha(t+r)}}\right)^{\frac{1}{2}}\left(\int_{\mathbf{R}}|f(t+r, t+r, x)-f(t+r, t, x)|^{2} m(\mathrm{~d} x)\right)^{\frac{1}{2}} \\
& \leq K_{U} r^{\frac{1}{\alpha(t+r)}-h(t)-\frac{1}{2}}\left(\int_{\mathbf{R}}|f(t+r, t+r, x)-f(t+r, t, x)|^{2} m(\mathrm{~d} x)\right)^{\frac{1}{2}} \\
& \leq K_{U} r^{\frac{1}{2}+\frac{1}{\alpha(t+r)}-h(t)} \text { with (H5) } \\
& \leq K_{U} r^{\frac{1}{2}+\frac{1}{d}-h_{+}},
\end{aligned}
$$

thus $\lim _{r \rightarrow 0} \sup _{t \in U} \delta_{2}(r, t)=0$.

$$
\delta_{3}(r, t) \leq \frac{C_{\alpha(t)}^{1 / \alpha(t)}}{2 r^{h(t)}}\left(\int_{\mathbf{R}}|f(t+r, t, x)|^{2} m(\mathrm{~d} x)\right)^{\frac{1}{2}}\left(\int_{\frac{K}{r}}\left(\frac{1}{y^{1 / \alpha(t+r)}}-\frac{1}{y^{1 / \alpha(t)}}\right)^{2} \mathrm{~d} y\right)^{\frac{1}{2}}
$$


Since the function $u \mapsto \alpha(u)$ is a $C^{1}$ function, $\forall \eta<\frac{1}{d}$,

$$
\begin{aligned}
\delta_{3}(r, t) & \leq \frac{K_{U}}{r^{h(t)}}\left(\int_{\mathbf{R}}|f(t+r, t, x)|^{2} m(\mathrm{~d} x)\right)^{\frac{1}{2}} K_{U} r^{\frac{1}{2}+\frac{1}{d}-\eta} \\
& \leq K_{U} r^{\frac{1}{2}+\frac{1}{d}-\eta-h_{+}} \text {with (M6) }
\end{aligned}
$$

thus $\lim _{r \rightarrow 0} \sup _{t \in U} \delta_{3}(r, t)=0$. Finally, $\lim _{r \rightarrow 0} \sup _{t \in U} \sqrt{\Delta_{1}(r, t)}=0$.

Let us now consider the last term $\Delta_{3}(r, t)$ :

$$
\Delta_{3}(r, t)=\frac{C_{\alpha(t)}^{2 / \alpha(t)} K^{1-\frac{2}{\alpha(t)}}}{\frac{2}{\alpha(t)}-1}\left(\frac{1}{r^{1+2(h(t)-1 / \alpha(t)}} \int_{\mathbf{R}}(f(t+r, t, x)-f(t, t, x))^{2} m(\mathrm{~d} x)-g(t)\right)
$$

thus, with (H4), $\lim _{r \rightarrow 0} \sup _{t \in U}\left|\Delta_{3}(r, t)\right|=0$

Lemma 5.13. Assume (M4), (M6), (M7),(H3), (H5), and let:

$$
\Delta(r, t)=: \frac{1}{r^{1+2(h(t)-1 / \alpha(t))}}\left(\frac{C_{\alpha(t)}^{1 / \alpha(t)} K^{\frac{1}{\alpha(t+r)}-\frac{1}{\alpha(t)}}\left(\frac{2}{\alpha(t+r)}-1\right) \int_{\mathbf{R}} f(t+r, t+r, x) f(t, t, x) m(\mathrm{~d} x)}{C_{\alpha(t+r)}^{1 / \alpha(t+r)} r^{\frac{1}{\alpha(t+r)}-\frac{1}{\alpha(t)}}\left(\frac{1}{\alpha(t+r)}+\frac{1}{\alpha(t)}-1\right) \int_{\mathbf{R}} f(t+r, t+r, x)^{2} m(\mathrm{~d} x)}-1\right)^{2} .
$$

Then:

$$
\lim _{r \rightarrow 0} \sup _{t \in U}|\Delta(r, t)|=0 .
$$

Proof. Since the function $t \mapsto \alpha(t)$ is a $C^{1}$ function, there exists $K_{U}>0$ such that

$$
\begin{gathered}
\left|\frac{C_{\alpha(t)}^{1 / \alpha(t)}}{C_{\alpha(t+r)}^{1 / \alpha(t+r)}}-1\right| \leq r K_{U}, \\
\left|K^{\frac{1}{\alpha(t+r)}-\frac{1}{\alpha(t)}}-1\right| \leq r K_{U},
\end{gathered}
$$

and

$$
\left|\frac{\frac{2}{\alpha(t+r)}-1}{\frac{1}{\alpha(t+r)}+\frac{1}{\alpha(t)}-1}-1\right| \leq r K_{U}
$$

Increasing $K_{U}$ if necessary, we also have, $\forall a>0$,

$$
\left|\frac{1}{r^{\frac{1}{\alpha(t+r)}-\frac{1}{\alpha(t)}}}-1\right| \leq r^{a} K_{U}
$$

For the last term, we write

$$
\frac{\int_{\mathbf{R}} f(t+r, t+r, x) f(t, t, x) m(\mathrm{~d} x)}{\int_{\mathbf{R}} f(t+r, t+r, x)^{2} m(\mathrm{~d} x)}-1=\Delta_{1}(r, t)+\Delta_{2}(r, t)
$$

where

$$
\Delta_{1}(r, t)=\frac{1}{\int_{\mathbf{R}} f(t+r, t+r, x)^{2} m(\mathrm{~d} x)}\left(\int_{\mathbf{R}} f(t+r, t+r, x)(f(t, t, x)-f(t+r, t, x)) m(\mathrm{~d} x)\right)
$$


and

$$
\Delta_{2}(r, t)=\frac{1}{\int_{\mathbf{R}} f(t+r, t+r, x)^{2} m(\mathrm{~d} x)}\left(\int_{\mathbf{R}} f(t+r, t+r, x)(f(t+r, t, x)-f(t+r, t+r, x)) m(\mathrm{~d} x)\right) .
$$

With (M7), we may choose $K_{U}$ such that

$$
\left|\Delta_{1}(r, t)\right| \leq K_{U} \int_{\mathbf{R}}|f(t+r, t+r, x)||f(t, t, x)-f(t+r, t, x)| m(\mathrm{~d} x) .
$$

Let $p \in(\alpha(t), 2), p \geq 1$ satisfying (H3), and $q$ such that $\frac{1}{p}+\frac{1}{q}=1$. Hölder inequality entails:

$$
\begin{aligned}
\left|\Delta_{1}(r, t)\right| & \leq K_{U}\left(\int_{\mathbf{R}}|f(t+r, t+r, x)|^{q} m(\mathrm{~d} x)\right)^{1 / q}\left(\int_{\mathbf{R}}|f(t, t, x)-f(t+r, t, x)|^{p} m(\mathrm{~d} x)\right)^{1 / p} \\
& \leq K_{U}\left(\int_{\mathbf{R}}|f(t+r, t, x)-f(t, t, x)|^{p} m(\mathrm{~d} x)\right)^{1 / p} \text { with (M4) and (M6) } \\
& \leq K_{U} r^{\frac{1}{p}+h(t)-\frac{1}{\alpha(t)}} \text { with (H3). }
\end{aligned}
$$

With (M6), (M7) and Cauchy-Schwarz inequality, we select $K_{U}$ such that

$$
\begin{aligned}
\left|\Delta_{2}(r, t)\right| & \leq K_{U}\left(\int_{\mathbf{R}}|f(t+r, t+r, x)-f(t+r, t, x)|^{2} m(\mathrm{~d} x)\right)^{\frac{1}{2}} \\
& \leq K_{U} r \text { with (H5). }
\end{aligned}
$$

Finally, since $h(t)+\frac{1}{p}-\frac{1}{\alpha(t)} \leq 1$

$$
\left|\frac{\int_{\mathbf{R}} f(t+r, t+r, x) f(t, t, x) m(\mathrm{~d} x)}{\int_{\mathbf{R}} f(t+r, t+r, x)^{2} m(\mathrm{~d} x)}-1\right| \leq K_{U} r^{h(t)+\frac{1}{p}-\frac{1}{\alpha(t)}} .
$$

Using the inequalities (5.12)-(5.16), we may find a constant $K_{U}$ such that for all $a>0$,

$$
|\Delta(r, t)| \leq \frac{1}{r^{1+2(h(t)-1 / \alpha(t))}} K_{U}\left(r^{2}+r^{2 a}+r^{2\left(h(t)+\frac{1}{p}-\frac{1}{\alpha(t)}\right)}\right) .
$$

Choosing $a \in\left(h(t)+\frac{1}{p}-\frac{1}{\alpha(t)}, 1\right)$, this entails:

$$
\begin{aligned}
|\Delta(r, t)| & \leq \frac{3}{r^{1+2(h(t)-1 / \alpha(t))}} K_{U} r^{2\left(h(t)+\frac{1}{p}-\frac{1}{\alpha(t)}\right)} \\
& \leq 3 K_{U} r^{\frac{2}{p}-1} .
\end{aligned}
$$

Since $\frac{2}{p}-1>0, \lim _{r \rightarrow 0} \sup _{t_{i} n U}|\Delta(r, t)|=0$ 
Lemma 5.14. Assuming (R1), (M4), (M5), (H1) one has:

$$
\begin{gathered}
\forall \varepsilon<\frac{1}{d}, \exists K_{U} \leq 1 \text { such that } \forall v \geq 1, \forall r \leq \varepsilon_{0}, \\
y \geq K_{U} \frac{v^{\frac{d}{1-\varepsilon d}}}{r} \Rightarrow \\
\Rightarrow t \in U, \sin ^{2}\left(\frac{v C_{\alpha(t+r)}^{1 / \alpha(t+r)} f(t+r, t+r, x)}{2 r^{h(t)} y^{1 / \alpha(t+r)}}-\frac{v C_{\alpha(t)}^{1 / \alpha(t)} f(t, t, x)}{2 r^{h(t)} y^{1 / \alpha(t)}}\right) \\
\geq \frac{1}{2}\left|\frac{v C_{\alpha(t+r)}^{1 / \alpha(t+r)} f(t+r, t+r, x)}{2 r^{h(t)} y^{1 / \alpha(t+r)}}-\frac{v C_{\alpha(t)}^{1 / \alpha(t)} f(t, t, x)}{2 r^{h(t)} y^{1 / \alpha(t)}}\right|^{2} .
\end{gathered}
$$

Proof. Let $\varepsilon<\frac{1}{d}$. We write $\frac{v C_{\alpha(t+r)}^{1 / \alpha(t+r)} f(t+r, t+r, x)}{2 r^{h(t)} y^{1 / \alpha(t+r)}}-\frac{v C_{\alpha(t)}^{1 / \alpha(t)} f(t, t, x)}{2 r^{h(t)} y^{1 / \alpha(t)}}=\kappa_{1}(r, t, v, x, y)+\kappa_{2}(r, t, v, x, y)$, with

$$
\kappa_{1}(r, t, v, x, y)=\frac{v}{2 r^{h(t)}}\left(\frac{C_{\alpha(t+r)}^{1 / \alpha(t+r)} f(t+r, t+r, x)}{y^{1 / \alpha(t+r)}}-\frac{C_{\alpha(t)}^{1 / \alpha(t)} f(t+r, t, x)}{y^{1 / \alpha(t)}}\right)
$$

and

$$
\kappa_{2}(r, t, v, x, y)=\frac{v C_{\alpha(t)}^{1 / \alpha(t)}}{2 r^{h(t)} y^{1 / \alpha(t)}}(f(t+r, t, x)-f(t, t, x)) .
$$

Using the finite-increments theorem,

$$
\begin{aligned}
\left|\kappa_{1}(r, t, v, x, y)\right| \leq & \frac{v}{2 r^{h(t)}} r\left(\sup _{a \in U}\left|\frac{K_{U}|f(t+r, a, x)|}{y^{1 / \alpha(a)}}\right|+\sup _{a \in U}\left|\frac{C_{\alpha(a)}^{1 / \alpha(a)}\left|f_{v}(t+r, a, x)\right|}{y^{1 / \alpha(a)}}\right|\right. \\
& \left.+\sup _{a \in U}\left|\frac{\left|\alpha^{\prime}(a)\right|}{\alpha^{2}(a)}\right| \ln y\left|\frac{C_{\alpha(a)}^{1 / \alpha(a)}|f(t+r, a, x)|}{y^{1 / \alpha(a)}}\right|\right) .
\end{aligned}
$$

For $y \geq 1$, conditions (M4) and (M5) imply

$$
\begin{aligned}
& \frac{K_{U}|f(t+r, a, x)|}{y^{1 / \alpha(a)}} \leq \frac{K_{U}}{y^{1 / d}}, \\
& \frac{K_{U}\left|f_{v}(t+r, a, x)\right|}{y^{1 / \alpha(a)}} \leq \frac{K_{U}}{y^{1 / d}},
\end{aligned}
$$

and

$$
\frac{\left|\alpha^{\prime}(a)\right|}{\alpha^{2}(a)}|\ln y| \frac{C_{\alpha(a)}^{1 / \alpha(a)}|f(t+r, a, x)|}{y^{1 / \alpha(a)}} \leq \frac{K_{U}|\ln y|}{y^{1 / d}}
$$

Finally,

$$
\begin{aligned}
\left|\kappa_{1}(r, t, v, x, y)\right| & \leq \frac{K_{U} v r^{1-h(t)}}{y^{1 / d}}(1+|\ln y|) \\
& \leq \frac{K_{U} v}{y^{1 / d-\varepsilon}} .
\end{aligned}
$$


Condition (H1) allows to estimate $\kappa_{2}(r, t, v, x, y)$ as follows:

$$
\left|\kappa_{2}(r, t, v, x, y)\right| \leq \frac{K_{U} v}{(r y)^{1 / \alpha(t)}} .
$$

Finally, $\forall K>0, \forall \varepsilon<\frac{1}{d}, \exists K_{U} \geq 1, \forall v \geq 1, \forall r<\varepsilon_{0}, \forall y \geq K_{U} \frac{v^{\frac{d}{1-\varepsilon d}}}{r}$,

$$
\left|\frac{v C_{\alpha(t+r)}^{1 / \alpha(t+r)} f(t+r, t+r, x)}{2 r^{h(t)} y^{1 / \alpha(t+r)}}-\frac{v C_{\alpha(t)}^{1 / \alpha(t)} f(t, t, x)}{2 r^{h(t)} y^{1 / \alpha(t)}}\right| \leq K .
$$

Lemma 5.15. Assuming (M4), (H3), (M6), (M7), (H4), (H5), there exist $\varepsilon_{0}$ and $K_{U}>0$ which does not depend on $t$ such that $\forall r<\varepsilon_{0}, \forall v \geq 1$ :

$$
N(v, t, r) \geq K_{U} v^{2+\frac{d}{1-\varepsilon d}\left(1-\frac{2}{c}\right)}
$$

where

$$
N(v, t, r)=: \int_{\mathbf{R}} \int_{\frac{K_{U} v}{r} \frac{d}{1-\varepsilon d}}\left|\frac{v C_{\alpha(t+r)}^{1 / \alpha(t+r)} f(t+r, t+r, x)}{2 r^{h(t)} y^{1 / \alpha(t+r)}}-\frac{v C_{\alpha(t)}^{1 / \alpha(t)} f(t, t, x)}{2 r^{h(t)} y^{1 / \alpha(t)}}\right|^{2} \mathrm{~d} y m(\mathrm{~d} x) .
$$

Proof. Expanding the square above, we may write

$$
N(v, t, r)=A_{1}(r, t) v^{2+\frac{d}{1-\varepsilon d}\left(1-\frac{2}{\alpha(t+r)}\right)}-A_{2}(r, t) v^{2+\frac{d}{1-\varepsilon d}}\left(1-\frac{1}{\alpha(t+r)}-\frac{1}{\alpha(t)}\right)+A_{3}(r, t) v^{2+\frac{d}{1-\varepsilon d}\left(1-\frac{2}{\alpha(t)}\right)},
$$

where

$$
\begin{gathered}
A_{1}(r, t)=\frac{C_{\alpha(t+r)}^{2 / \alpha(t+r)}\left(K_{U}\right)^{1-\frac{2}{\alpha(t+r)}}}{4\left(\frac{2}{\alpha(t+r)}-1\right) r^{1+2\left(h(t)-\frac{1}{\alpha(t+r)}\right)}} \int_{\mathbf{R}}|f(t+r, t+r, x)|^{2} m(\mathrm{~d} x), \\
A_{2}(r, t)=\frac{C_{\alpha(t+r)}^{1 / \alpha(t+r)} C_{\alpha(t)}^{1 / \alpha(t)}\left(K_{U}\right)^{1-\frac{1}{\alpha(t+r)}-\frac{1}{\alpha(t)}}}{2\left(\frac{1}{\alpha(t+r)}+\frac{1}{\alpha(t)}-1\right) r^{1+2 h(t)-\frac{1}{\alpha(t+r)}-\frac{1}{\alpha(t)}}} \int_{\mathbf{R}} f(t+r, t+r, x) f(t, t, x) m(\mathrm{~d} x),
\end{gathered}
$$

and

$$
A_{3}(r, t)=\frac{C_{\alpha(t)}^{2 / \alpha(t)}\left(K_{U}\right)^{1-\frac{2}{\alpha(t)}}}{4\left(\frac{2}{\alpha(t)}-1\right) r^{1+2\left(h(t)-\frac{1}{\alpha(t)}\right)}} \int_{\mathbf{R}}|f(t, t, x)|^{2} m(\mathrm{~d} x) .
$$

We obtain

$$
N(v, t, r)=v^{2+\frac{d}{1-\varepsilon d}\left(1-\frac{2}{\alpha(t)}\right)}\left(A_{1}(r, t)\left(v^{\frac{2 d}{1-\varepsilon d}\left(\frac{1}{\alpha(t)}-\frac{1}{\alpha(t+r)}\right)}\right)^{2}-A_{2}(r, t)\left(v^{\frac{2 d}{1-\varepsilon d}\left(\frac{1}{\alpha(t)}-\frac{1}{\alpha(t+r)}\right)}\right)+A_{3}(r, t)\right) .
$$

Let $P(r, t, X)=A_{1}(r, t) X^{2}-A_{2}(r, t) X+A_{3}(r, t)$. Write:

$$
P(r, t, X)=P(r, t, X)-P\left(r, t, \frac{A_{2}(r, t)}{2 A_{1}(r, t)}\right)+P\left(r, t, \frac{A_{2}(r, t)}{2 A_{1}(r, t)}\right)-P(r, t, 1)+P(r, t, 1) .
$$

Since $P\left(\frac{A_{2}(r, t)}{2 A_{1}(r, t)}\right)$ is the minimum of $P$,

$$
P(r, t, X) \geq P\left(r, t, \frac{A_{2}(r, t)}{2 A_{1}(r, t)}\right)-P(r, t, 1)+P(r, t, 1) .
$$


Note that $P(r, t, 1)=N(1, t, r)$, thus Lemma 5.12 entails that there exists a positive function $l$ such that $\lim _{r \rightarrow 0} P(r, t, 1)=l(t)$. For $P\left(r, t, \frac{A_{2}(r, t)}{2 A_{1}(r, t)}\right)-P(r, t, 1)$, we use Lemma 5.13. With the same notations,

$$
\begin{aligned}
\left|P\left(r, t, \frac{A_{2}(r, t)}{2 A_{1}(r, t)}\right)-P(r, t, 1)\right| & =\left|A_{1}(r, t)\right| r^{1+2\left(h(t)-\frac{1}{\alpha(t)}\right)} \Delta(r, t) \\
& \leq K_{U} \Delta(r, t)
\end{aligned}
$$

thus $\lim _{r \rightarrow 0} \sup _{t \in U}\left|P\left(r, t, \frac{A_{2}(r, t)}{2 A_{1}(r, t)}\right)-P(r, t, 1)\right|=0$. As a consequence, there exist a positive constant $K_{U}$ and $\varepsilon_{0}>0$ such that for all $x \in \mathbf{R}$, all $r \in\left(0, \varepsilon_{0}\right)$ and all $t_{i} n U, P(r, t, x) \geq K_{U}$. We obtain $N(v, t, r) \geq v^{2+\frac{d}{1-\varepsilon d}\left(1-\frac{2}{\alpha(t)}\right)} K_{U}$ for all $v \in \mathbf{R}$ and $r \in\left(0, \varepsilon_{0}\right)$. Since $\alpha(t)>c, N(v, t, r) \geq K_{U} v^{2+\frac{d}{1-\varepsilon d}\left(1-\frac{2}{c}\right)}$

We now proceed to the Proof of Theorem 3.2.

Let $\gamma>h(t)$ and $x>0$.

$$
\mathrm{P}\left(\frac{r^{\gamma}}{|Y(t+r)-Y(t)|}>x\right)=\mathrm{P}\left(|Y(t+r)-Y(t)|<\frac{r^{\gamma}}{x}\right) .
$$

Applying Proposition 4.10, there exists $\varepsilon_{0}>0$ such that

$$
\sup _{r \in B\left(0, \varepsilon_{0}\right)} \int_{0}^{+\infty} \varphi_{\frac{Y(t+r)-Y(t)}{r^{h(t)}}}(v) \mathrm{d} v<+\infty .
$$

Thus with Proposition 4.9, there exists $K_{U}>0$ such that

$$
\mathrm{P}\left(|Y(t+r)-Y(t)|<\frac{r^{\gamma}}{x}\right) \leq K_{U} \frac{r^{\gamma-h(t)}}{x} .
$$

Let $r_{n}=\frac{1}{n^{\eta}}$ with $\eta(\gamma-h(t))>1 . \forall x>0, \sum_{n} \mathrm{P}\left(\frac{r_{n}^{\gamma}}{\left|Y\left(t+r_{n}\right)-Y(t)\right|}>x\right)<+\infty$. Borel Cantelli lemma entails that, almost surely, $\lim _{n \rightarrow+\infty} \frac{\left|Y\left(t+r_{n}\right)-Y(t)\right|}{r_{n}^{\gamma}}=+\infty$. As a consequence, almost surely, $\limsup _{r \rightarrow 0} \frac{|Y(t+r)-Y(t)|}{r^{\gamma}}=+\infty$, and

$$
\mathcal{H}_{t} \leq h(t)
$$

\subsection{Proof of Theorem 3.5}

We want to apply Theorem 3.1 with $f(t, u, x)=\mathbf{1}_{[0, t]}(x)$. Let us show that conditions (R1), (M1), (M2) and (H2) are satisfied.

- (R1) The family of functions $v \rightarrow f(t, v, x)$ is differentiable for all $(v, t)$ in $(0,1)^{2}$ and almost all $x$ in $E$. The derivatives of $f$ with respect to $u$ vanish.

- (M1)

$$
|f(t, w, x)|^{\alpha(w)}=\mathbf{1}_{[0, t]}(x)
$$

thus, for all $\delta>0$, all $t \in(0,1)$,

$$
\int_{\mathbf{R}}\left[\sup _{w \in(0,1)}\left(|f(t, w, x)|^{\alpha(w)}\right)\right]^{1+\delta} \mathrm{d} x=t
$$

and (M1) holds. 
- (M2) $f_{u}^{\prime}=0$ thus (M2) holds.

- $(\mathrm{H} 2)$

$$
\begin{aligned}
\frac{1}{r^{h(t) \alpha(t)}} \int_{\mathbf{R}}|f(t+r, t, x)-f(t, t, x)|^{\alpha(t)} m(\mathrm{~d} x) & =\frac{1}{r} \int_{t}^{t+r} \mathrm{~d} x \\
& =1,
\end{aligned}
$$

thus (H2) holds.

From Theorem 3.1, we get that

$$
\mathrm{E}\left[|Y(t+\varepsilon)-Y(t)|^{\eta}\right] \sim \varepsilon^{\frac{\eta}{\alpha(t)}} \mathrm{E}\left[\left|Y_{t}^{\prime}(1)\right|^{\eta}\right] .
$$

Since $Y_{t}^{\prime}(1)$ is an $S_{\alpha(t)}(1,0,0)$ random variable, Property 1.2 .17 of [21] allows to conclude.

\subsection{Proof of Theorem 3.6}

We want to apply Theorem 3.2 with $f(t, u, x)=\mathbf{1}_{[0, t]}(x)$ and $h(t)=\frac{1}{\alpha(t)}$ in order to obtain the inequality. Let us show that the conditions (M4), (M5), (M6), (M7), (H1), (H3), (H4) and (H5) are satisfied.

- (M4) Obvious.

- (M5) Obvious.

- (H1) $\forall v \in U, \forall u \in U, \forall x \in \mathbf{R}$,

$$
\begin{aligned}
\frac{1}{|v-u|^{h(u)-1 / \alpha(u)}}|f(v, u, x)-f(u, u, x)| & =\mathbf{1}_{[u, v]}(x) \\
& \leq 1
\end{aligned}
$$

thus (H1) holds.

- (H3) $\forall v \in U, \forall u \in U$,

$$
\begin{aligned}
\frac{1}{|v-u|^{1+p\left(h(u)-\frac{1}{\alpha(u)}\right)}} \int_{\mathbf{R}}|f(v, u, x)-f(u, u, x)|^{p} m(\mathrm{~d} x) & =\frac{1}{|v-u|} \int_{\mathbf{R}}\left|\mathbf{1}_{[u, v]}(x)\right| \\
& =1
\end{aligned}
$$

thus (H3) holds.

- (M6) $\forall v \in U, \forall u \in U$,

$$
\int_{\mathbf{R}}|f(v, u, x)|^{2} m(\mathrm{~d} x)=v
$$

thus (M6) holds $(U=(0,1))$.

- (M7) Since $t \in(0,1)$ (in particular $t \neq 0$ ), one can choose $U$ such that $\inf _{v \in U} v>0$ thus (M7) holds.

- (H4)

$$
\begin{aligned}
\frac{1}{\left.r^{1+2(h(t)-1 / \alpha(t)}\right)} \int_{\mathbf{R}}(f(t+r, t, x)-f(t, t, x))^{2} m(\mathrm{~d} x) & =\frac{1}{r} \int_{\mathbf{R}} \mathbf{1}_{[t, t+r]}(x) \mathrm{d} x \\
& =1
\end{aligned}
$$

thus (H4) holds. 
- (H5) $\forall v \in U, \forall u \in U$,

$$
\frac{1}{|v-u|^{2}} \int_{\mathbf{R}}|f(v, v, x)-f(v, u, x)|^{2} m(\mathrm{~d} x)=0
$$

thus (H5) holds.

\subsection{Proof of Theorem 3.3}

We want to apply Theorem 3.1 with $f(t, u, x)=|t-x|^{H(u)-\frac{1}{\alpha(u)}}-|x|^{H(u)-\frac{1}{\alpha(u)}}$. Let us show that conditions (R1), (M1), (M2), (M3) and (H2) are satisfied.

- (R1) The family of functions $u \rightarrow f(t, u, x)$ is differentiable for all $(u, t)$ in a neighbourhood of $t_{0}$ and almost all $x$ in $E$. The derivatives of $f$ with respect to $u$ read:

$$
f_{u}^{\prime}(t, w, x)=\left(h^{\prime}(w)+\frac{\alpha^{\prime}(w)}{\alpha^{2}(w)}\right)\left[(\log |t-x|)|t-x|^{h(w)-1 / \alpha(w)}-(\log |x|)|x|^{h(w)-1 / \alpha(w)}\right] .
$$

- (M1) In [6], it is shown that, given $t_{0} \in \mathbf{R}$, one may choose $\varepsilon>0$ small enough and numbers $a, b, h_{-}, h_{+}$ with $0<a<\alpha(w)<b<2,0<h_{-}<h(w)<h_{+}<1$ and $\frac{a}{b}\left(\frac{1}{a}-\frac{1}{b}\right)<h_{-}-\left(\frac{1}{a}-\frac{1}{b}\right)<h_{-}<h_{+}<$ $h_{+}+\left(\frac{1}{a}-\frac{1}{b}\right)<1-\left(\frac{1}{a}-\frac{1}{b}\right)$ such that, for all $t$ and $w$ in $U:=\left(t_{0}-\varepsilon, t_{0}+\varepsilon\right)$ and all real $x$ :

$$
|f(t, w, x)|,\left|f_{t_{0}}^{\prime}(t, w, x)\right| \leq k_{1}(t, x)
$$

where

$$
k_{1}(t, x)= \begin{cases}c_{1} \max \left\{1,|t-x|^{h_{-}-1 / a}+|x|^{h_{--1 / a}}\right\} & \left(|x| \leq 1+2 \max _{t \in U}|t|\right) \\ c_{2}|x|^{h_{+}-1 / b-1} & \left(|x|>1+2 \max _{t \in U}|t|\right)\end{cases}
$$

for appropriately chosen constants $c_{1}$ and $c_{2}$. One has, for all $\delta>0$,

$$
\begin{aligned}
\int_{\mathbf{R}}\left[\sup _{w \in U}|f(t, w, x)|^{\alpha(w)}\right]^{1+\delta} r(x)^{\delta} \mathrm{d} x \leq & \int_{\mathbf{R}}\left(k_{1}(t, x)^{a}+k_{1}(t, x)^{b}\right)^{1+\delta} r(x)^{\delta} \mathrm{d} x \\
\leq & K_{\delta} \int_{\mathbf{R}} k_{1}(t, x)^{a(1+\delta)} r(x)^{\delta} \mathrm{d} x \\
& +K_{\delta} \int_{\mathbf{R}} k_{1}(t, x)^{b(1+\delta)} r(x)^{\delta} \mathrm{d} x .
\end{aligned}
$$

Let us study $\int_{\mathbf{R}} k_{1}(t, x)^{p(1+\delta)} r(x)^{\delta} \mathrm{d} x$, where $p=a$ or $p=b$.

$$
\begin{aligned}
\int_{\mathbf{R}} k_{1}(t, x)^{p(1+\delta)} r(x)^{\delta} \mathrm{d} x & =\frac{\pi^{2 \delta}}{3^{\delta}} \sum_{j=0}^{+\infty}(j+1)^{2 \delta} \int_{j}^{j+1}\left(k_{1}(t,-x)^{p(1+\delta)}+k_{1}(t, x)^{p(1+\delta)}\right) \mathrm{d} x \\
& =\frac{\pi^{2 \delta}}{3^{\delta}} \sum_{j=0}^{+\infty}(j+1)^{2 \delta} \int_{j}^{j+1}\left(k_{1}(-t, x)^{p(1+\delta)}+k_{1}(t, x)^{p(1+\delta)}\right) \mathrm{d} x
\end{aligned}
$$

We consider now $\int_{j}^{j+1} k_{1}( \pm t, x)^{p(1+\delta)} \mathrm{d} x$. There exists $K_{p, \delta}>0$ such that, for all real $x$ such that $|x| \leq$ $1+2 \max _{t \in U}|t|:$

$$
k_{1}( \pm t, x)^{p(1+\delta)} \leq K_{p, \delta}\left(1+| \pm t-x|^{p(1+\delta)\left(h_{-}-1 / a\right)}+|x|^{p(1+\delta)\left(h_{-}-1 / a\right)}\right),
$$


and for all real $x$ such that $|x|>1+2 \max _{t \in U}|t|$,

$$
k_{1}( \pm t, x)^{p(1+\delta)} \leq K_{p, \delta}|x|^{p(1+\delta)\left(h_{+}-1 / b-1\right)} .
$$

Let $\left.j_{0}=\left[1+2 \max _{t \in U}|t|\right)\right]$. For $j<j_{0}$,

$$
\int_{j}^{j+1} k_{1}( \pm t, x)^{p(1+\delta)} \mathrm{d} x \leq K_{p, \delta}\left(1+\int_{j}^{j+1}| \pm t-x|^{p(1+\delta)\left(h_{-}-1 / a\right)} \mathrm{d} x+\int_{j}^{j+1}|x|^{p(1+\delta)\left(h_{-}-1 / a\right)} \mathrm{d} x\right) .
$$

Choose $\delta$ such that $p(1+\delta)\left(h_{-}-1 / a\right)>-1$ (we show below that such a $\delta$ exists).

Then

$$
\begin{aligned}
\int_{j}^{j+1}| \pm t-x|^{p(1+\delta)\left(h_{-}-1 / a\right)} \mathrm{d} x & =\int_{ \pm t-j-1}^{ \pm t-j}|u|^{p(1+\delta)\left(h_{-}-1 / a\right)} \mathrm{d} u \\
& \leq \frac{| \pm t-j|^{1+p(1+\delta)\left(h_{-}-1 / a\right)}+| \pm t-j-1|^{1+p(1+\delta)\left(h_{-}-1 / a\right)}}{1+p(1+\delta)\left(h_{-}-1 / a\right)} \\
& \leq K_{U}|t|^{1+p(1+\delta)\left(h_{-}-1 / a\right)}|1+j|^{1+p(1+\delta)\left(h_{-}-1 / a\right)} \\
& \leq K_{U}(1+j)^{1+p(1+\delta)\left(h_{-}-1 / a\right)}
\end{aligned}
$$

where $K_{U}>0$ depends on $U$ and may have changed from line to line. We deduce:

$$
\int_{j}^{j+1} k_{1}( \pm t, x)^{p(1+\delta)} \mathrm{d} x \leq K_{U}\left(1+j^{1+p(1+\delta)\left(h_{-}-1 / a\right)}\right) .
$$

For $j=j_{0}$,

$$
\begin{aligned}
\int_{j_{0}}^{j_{0}+1} k_{1}( \pm t, x)^{p(1+\delta)} \mathrm{d} x & \leq K_{U}\left|j_{0}\right|^{1+p(1+\delta)\left(h_{-}-1 / a\right)}+K_{U} \int_{j_{0}}^{j_{0}+1}|x|^{p(1+\delta)\left(h_{+}-1 / b-1\right)} \mathrm{d} x \\
& \leq K_{U}
\end{aligned}
$$

For $j>j_{0}$,

$$
\begin{aligned}
\int_{j}^{j+1} k_{1}( \pm t, x)^{p(1+\delta)} \mathrm{d} x & \leq K_{U} \int_{j}^{j+1}|x|^{p(1+\delta)\left(h_{+}-1 / b-1\right)} \mathrm{d} x \\
& \leq K_{U} j^{p(1+\delta)\left(h_{+}-1 / b-1\right)}
\end{aligned}
$$

Finally,

$$
\begin{gathered}
\sup _{t \in U} \int_{\mathbf{R}} k_{1}(t, x)^{p(1+\delta)} r(x)^{\delta} \mathrm{d} x \leq \\
K_{U}\left(1+\sum_{j=0}^{j_{0}-1} j^{2 \delta}\left(1+j^{1+p(1+\delta)\left(h_{-}-1 / a\right)}\right)\right) \\
+K_{U} \sum_{j=j_{0}+1}^{\infty} j^{2 \delta+p(1+\delta)\left(h_{+}-1 / b-1\right)}
\end{gathered}
$$

To conclude, we need to show that we may chose $\delta>\frac{b}{a}-1$ such that $p(1+\delta)\left(h_{-}-1 / a\right)>-1$ and $2 \delta+p(1+\delta)\left(h_{+}-1 / b-1\right)<-1$, for $p=a$ and $p=b$. We consider several cases. 
First case: $h_{-}-\frac{1}{a} \geq 0$ and $h_{+}-\frac{1}{b}-1 \leq-\frac{2}{a}$.

Let $\delta>\frac{b}{a}-1$. One has $p(1+\delta)\left(h_{-}-\frac{1}{a}\right) \geq 0>-1$. We consider $1+2 \delta+p(1+\delta)\left(h_{+}-1 / b-1\right)$.

$$
\begin{aligned}
1+2 \delta+p(1+\delta)\left(h_{+}-1 / b-1\right) & \leq 1+2 \delta-\frac{2}{a} p(1+\delta) \\
& =1-\frac{2 p}{a}+2 \delta\left(1-\frac{p}{a}\right) .
\end{aligned}
$$

Since $1-\frac{2 p}{a}<0$ and $1-\frac{p}{a} \leq 0,1+2 \delta+p(1+\delta)\left(h_{+}-1 / b-1\right)<0$.

Second case: $h_{-}-\frac{1}{a} \geq 0$ and $h_{+}-\frac{1}{b}-1>-\frac{2}{a}$.

Let $\delta \in\left(\frac{b}{a}-1, \frac{\frac{1}{b}-\frac{1}{a}+1-h_{+}}{\frac{2}{a}-\frac{1}{b}-1+h_{+}}\right)$. One has $p(1+\delta)\left(h_{-}-\frac{1}{a}\right) \geq 0>-1$. We consider $1+2 \delta+p(1+\delta)\left(h_{+}-1 / b-1\right)$.

For $p=a$ :

$$
\begin{aligned}
1+2 \delta+p(1+\delta)\left(h_{+}-1 / b-1\right) & =a \delta\left(\frac{2}{a}+h_{+}-\frac{1}{b}-1\right)+a\left(h_{+}-\frac{1}{b}-1+\frac{1}{a}\right) \\
& <a\left(\frac{1}{b}-\frac{1}{a}+1-h_{+}\right)+a\left(h_{+}-\frac{1}{b}-1+\frac{1}{a}\right) \\
& =0 .
\end{aligned}
$$

For $p=b$ :

$$
1+2 \delta+p(1+\delta)\left(h_{+}-1 / b-1\right)=b \delta\left(\frac{1}{b}+h_{+}-1\right)+b\left(h_{+}-1\right) .
$$

If $\frac{1}{b}+h_{+}-1 \leq 0$, then $b \delta\left(\frac{1}{b}+h_{+}-1\right)+b\left(h_{+}-1\right)<0$. Else

$$
\begin{aligned}
b \delta\left(\frac{1}{b}+h_{+}-1\right)+b\left(h_{+}-1\right) & <b \frac{\frac{1}{b}-\frac{1}{a}+1-h_{+}}{\frac{2}{a}-\frac{1}{b}-1+h_{+}}\left(\frac{1}{b}+h_{+}-1\right)+b\left(h_{+}-1\right) \\
& =\frac{b}{\frac{2}{a}-\frac{1}{b}-1+h_{+}}\left(\frac{1}{a}-\frac{1}{b}\right)\left(h_{+}-1-\frac{1}{b}\right) \\
& <0 .
\end{aligned}
$$

Third case: $h_{-}-\frac{1}{a}<0$ and $h_{+}-\frac{1}{b}-1 \leq-\frac{2}{a}$.

Let $\delta \in\left(\frac{b}{a}-1, \frac{a h_{-}+\frac{a}{b}-1}{1-a h_{-}}\right)$.

For $p=a$ :

$$
\begin{aligned}
1+p(1+\delta)\left(h_{-}-\frac{1}{a}\right) & =a h_{-}+\delta\left(a h_{-}-1\right) \\
& >a h_{-}+\left(a h_{-}-1\right) \frac{a h_{-}+\frac{a}{b}-1}{1-a h_{-}} \\
& =a h_{-}+1-\frac{a}{b}-a h_{-} \\
& >0,
\end{aligned}
$$


and

$$
\begin{aligned}
1+2 \delta+p(1+\delta)\left(h_{+}-1 / b-1\right) & =a \delta\left(\frac{2}{a}+h_{+}-\frac{1}{b}-1\right)+a\left(h_{+}-\frac{1}{b}-1+\frac{1}{a}\right) \\
& \leq a\left(h_{+}-\frac{1}{b}-1+\frac{1}{a}\right) \\
& \leq-1 \\
& <0 .
\end{aligned}
$$

For $p=b$ :

$$
\begin{aligned}
1+p(1+\delta)\left(h_{-}-\frac{1}{a}\right) & =b\left(h_{-}-\frac{1}{a}+\frac{1}{b}\right)+b \delta\left(h_{-}-\frac{1}{a}\right) \\
& >b\left(h_{-}-\frac{1}{a}+\frac{1}{b}\right)+b\left(h_{-}-\frac{1}{a}\right) \frac{a h_{-}+\frac{a}{b}-1}{1-a h_{-}} \\
& =b\left(h_{-}-\frac{1}{a}+\frac{1}{b}\right)+b\left(\frac{1}{a}-\frac{1}{b}-h_{-}\right) \\
& =0
\end{aligned}
$$

and

$$
\begin{gathered}
1+2 \delta+p(1+\delta)\left(h_{+}-1 / b-1\right)=b \delta\left(\frac{1}{b}+h_{+}-1\right)+b\left(h_{+}-1\right) \\
\leq b \delta\left(\frac{2}{b}-\frac{2}{a}\right)+b\left(h_{+}-1\right) \\
<0
\end{gathered}
$$

Fourth case: $h_{-}-\frac{1}{a}<0$ and $h_{+}-\frac{1}{b}-1>-\frac{2}{a}$.

Let $\delta \in\left(\frac{b}{a}-1, \min \left(\frac{a h_{-}+\frac{a}{b}-1}{1-a h_{-}}, \frac{\frac{1}{b}-\frac{1}{a}+1-h_{+}}{\frac{2}{a}-\frac{1}{b}-1+h_{+}}\right)\right)$.

For $p=a$ :

$$
\begin{aligned}
1+p(1+\delta)\left(h_{-}-\frac{1}{a}\right) & =a h_{-}+\delta\left(a h_{-}-1\right) \\
& >a h_{-}+\left(a h_{-}-1\right) \frac{a h_{-}+\frac{a}{b}-1}{1-a h_{-}} \\
& =a h_{-}+1-\frac{a}{b}-a h_{-} \\
& >0,
\end{aligned}
$$


and

$$
\begin{aligned}
1+2 \delta+p(1+\delta)\left(h_{+}-1 / b-1\right) & =a \delta\left(\frac{2}{a}+h_{+}-\frac{1}{b}-1\right)+a\left(h_{+}-\frac{1}{b}-1+\frac{1}{a}\right) \\
& >a\left(\frac{1}{b}-\frac{1}{a}+1-h_{+}\right)+a\left(h_{+}-\frac{1}{b}-1+\frac{1}{a}\right) \\
& =0 .
\end{aligned}
$$

For $p=b$ :

$$
\begin{aligned}
1+p(1+\delta)\left(h_{-}-\frac{1}{a}\right) & =b\left(h_{-}-\frac{1}{a}+\frac{1}{b}\right)+b \delta\left(h_{-}-\frac{1}{a}\right) \\
& >b\left(h_{-}-\frac{1}{a}+\frac{1}{b}\right)+b\left(h_{-}-\frac{1}{a}\right) \frac{a h_{-}+\frac{a}{b}-1}{1-a h_{-}} \\
& =b\left(h_{-}-\frac{1}{a}+\frac{1}{b}\right)+b\left(\frac{1}{a}-\frac{1}{b}-h_{-}\right) \\
& =0
\end{aligned}
$$

and

$$
1+2 \delta+p(1+\delta)\left(h_{+}-1 / b-1\right)=b \delta\left(\frac{1}{b}+h_{+}-1\right)+b\left(h_{+}-1\right)
$$

If $\frac{1}{b}+h_{+}-1 \leq 0$, then $1+2 \delta+p(1+\delta)\left(h_{+}-1 / b-1\right)<0$, else

$$
\begin{aligned}
b \delta\left(\frac{1}{b}+h_{+}-1\right)+b\left(h_{+}-1\right) & <b\left(\frac{\frac{1}{b}-\frac{1}{a}+1-h_{+}}{\frac{2}{a}-\frac{1}{b}-1+h_{+}}\right)\left(\frac{1}{b}+h_{+}-1\right)+b\left(h_{+}-1\right) \\
& =\frac{b}{\frac{2}{a}-\frac{1}{b}-1+h_{+}}\left(\frac{1}{a}-\frac{1}{b}\right)\left(h_{+}-1-\frac{1}{b}\right) \\
& <0 .
\end{aligned}
$$

- (M2) is obtained with (5.17) for the same reason as in (M1).

- (M3) For $j$ large enough $\left(j>j^{*}\right)$,

$$
\begin{aligned}
&|f(t, w, x) \log (r(x))|^{\alpha(w)} \leq K_{1}\left|k_{1}(t, x)\right|^{\alpha(w)} \\
&+K_{2} \sum_{j=j^{*}}^{+\infty}|f(t, w, x)|^{\alpha(w)}(\log (j))^{d} \mathbf{1}_{[-j,-j+1[\cup[j-1, j[}(x) . \\
&|f(t, w, x)|^{\alpha(w)} \mathbf{1}_{[-j,-j+1[\cup[j-1, j[}(x) \leq K_{2} \frac{1}{|x|^{a\left(1+1 / b-h_{+}\right)}} \mathbf{1}_{[-j,-j+1[\cup[j-1, j[}(x)
\end{aligned}
$$


( $K_{2}$ may have changed from line to line). Thus

$$
\begin{aligned}
{\left[\sup _{w \in U}\left[|f(t, w, x) \log (r(x))|^{\alpha(w)}\right]\right]^{1+\delta} r(x)^{\delta} \leq } & K\left|k_{1}(t, x)\right|^{a(1+\delta)} r(x)^{\delta}+K\left|k_{1}(t, x)\right|^{b(1+\delta)} r(x)^{\delta} \\
& +K \sum_{j=j^{*}}^{+\infty} \frac{j^{2 \delta}(\log (j))^{d}}{|x|^{a(1+\delta)\left(1+1 / b-h_{+}\right)}} \mathbf{1}_{[-j,-j+1[\cup[j-1, j[}(x) .
\end{aligned}
$$

Let $\delta>\frac{b}{a}-1$ be such that (M1) holds. Since $2 \delta+a(1+\delta)\left(h_{+}-1-\frac{1}{b}\right)<-1$, (M3) holds.

- $(\mathrm{H} 2)$

$$
\frac{1}{r^{H(t) \alpha(t)}} \int_{\mathbf{R}}|f(t+r, t, x)-f(t, t, x)|^{\alpha(t)} m(\mathrm{~d} x)=\int_{\mathbf{R}}|| 1-\left.x\right|^{H(t)-\frac{1}{\alpha(t)}}-\left.|x|^{H(t)-\frac{1}{\alpha(t)}}\right|^{\alpha(t)} \mathrm{d} x
$$

so (H2) holds.

From Theorem 3.1, we obtain that

$$
\mathrm{E}\left[|Y(t+\varepsilon)-Y(t)|^{\eta}\right] \sim \varepsilon^{\eta H(t)} \mathrm{E}\left[\left|Y_{t}^{\prime}(1)\right|^{\eta}\right] .
$$

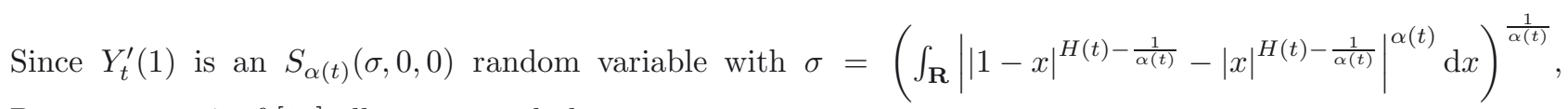
Property 1.2.17 of [21] allows to conclude.

\subsection{Proof of Theorem 3.4}

We want to apply Theorems 3.2 with $f(t, u, x)=|t-x|^{H(u)-\frac{1}{\alpha(u)}}-|x|^{H(u)-\frac{1}{\alpha(u)}}$ in order to obtain the inequality. Let us show that conditions (M4), (M5), (M6), (M7), (H1), (H3), (H4) and (H5) are satisfied.

- (M4) Since $H(t)-\frac{1}{\alpha(t)} \geq 0$, (M4) holds.

- (M5) We also use the fact that $H(t)-\frac{1}{\alpha(t)} \geq 0$ in order to prove that (M5) holds.

- (H1) $\forall v \in U, \forall u \in U, \forall x \in \mathbf{R}$,

$$
\begin{aligned}
\frac{1}{|v-u|^{h(u)-1 / \alpha(u)}}|f(v, u, x)-f(u, u, x)| & =\frac{1}{|v-u|^{H(u)-1 / \alpha(u)}}|| v-\left.x\right|^{H(u)-\frac{1}{\alpha(u)}}-|u-x|^{H(u)-\frac{1}{\alpha(u)}} \mid \\
& \leq 1
\end{aligned}
$$

thus (H1) holds.

- (H3) $\forall v \in U, \forall u \in U$,

$$
\frac{1}{|v-u|^{1+p\left(h(u)-\frac{1}{\alpha(u)}\right)}} \int_{\mathbf{R}}|f(v, u, x)-f(u, u, x)|^{p} m(\mathrm{~d} x)=\int_{\mathbf{R}}|| 1-\left.x\right|^{H(u)-\frac{1}{\alpha(u)}}-\left.|x|^{H(u)-\frac{1}{\alpha(u)}}\right|^{p} \mathrm{~d} x
$$

so (H3) holds.

- (M6) $\forall v \in U, \forall u \in U$,

$$
\int_{\mathbf{R}}|f(v, u, x)|^{2} m(\mathrm{~d} x)=v^{1+2\left(H(u)-\frac{1}{\alpha(u)}\right)} \int_{\mathbf{R}}|| 1-\left.x\right|^{H(u)-\frac{1}{\alpha(u)}}-\left.|x|^{H(u)-\frac{1}{\alpha(u)}}\right|^{2} \mathrm{~d} x
$$

thus (M6) holds. 
- (M7) For $t \neq 0$, one can choose $U$ such that $\inf _{v \in U} v^{1+2\left(H(v)-\frac{1}{\alpha(v)}\right)}>0$ thus (M7) holds.

- (H4)

$$
\frac{1}{\left.r^{1+2(h(t)-1 / \alpha(t)}\right)} \int_{\mathbf{R}}(f(t+r, t, x)-f(t, t, x))^{2} m(\mathrm{~d} x)=\int_{\mathbf{R}}|| 1-\left.x\right|^{H(t)-\frac{1}{\alpha(t)}}-\left.|x|^{H(t)-\frac{1}{\alpha(t)}}\right|^{2} \mathrm{~d} x
$$

thus, choosing $g(t)=\int_{\mathbf{R}}|| 1-\left.x\right|^{H(t)-\frac{1}{\alpha(t)}}-\left.|x|^{H(t)-\frac{1}{\alpha(t)}}\right|^{2} \mathrm{~d} x$, (H4) holds.

- (H5) $\forall v \in U, \forall u \in U$,

$$
\begin{array}{r}
\frac{1}{|v-u|^{2}} \int_{\mathbf{R}}|f(v, v, x)-f(v, u, x)|^{2} m(\mathrm{~d} x) \\
=\frac{1}{|v-u|^{2}} \int_{\mathbf{R}}|| v-\left.x\right|^{H(v)-\frac{1}{\alpha(v)}}-|v-x|^{H(u)-\frac{1}{\alpha(u)}}-|x|^{H(v)-\frac{1}{\alpha(v)}}+\left.|x|^{H(u)-\frac{1}{\alpha(u)}}\right|^{2} \mathrm{~d} x
\end{array}
$$

thus (H5) holds

\subsection{Proof of Theorem 3.8}

Recall that $r(x)=\sum_{j=1}^{+\infty} 2^{j+1} \mathbf{1}_{[-j,-j+1[\cup[j-1, j[}(x)$ and $f(t, w, x)=\mathrm{e}^{-\lambda(x-t)} \mathbf{1}_{[t,+\infty)}(x)$.

- $(\mathrm{R} 1) f_{v}^{\prime}(t, w, x) \equiv 0$.

- (M1)

$$
\begin{gathered}
{\left[\sup _{w \in U}|f(t, w, x)|^{\alpha(w)}\right]^{1+\delta} \leq \mathrm{e}^{-\lambda c(1+\delta)(x-t)} \mathbf{1}_{[t,+\infty)}(x),} \\
{\left[\sup _{w \in U}|f(t, w, x)|^{\alpha(w)}\right]^{1+\delta} r(x)^{\delta} \leq \sum_{j=1}^{+\infty} 2^{(j+1) \delta} \mathrm{e}^{-\lambda c(1+\delta)(x-t)} \mathbf{1}_{([-j,-j+1[\cup[j-1, j[) \cap[t,+\infty)}(x) .}
\end{gathered}
$$

We write $U=(t-\epsilon, t+\epsilon)$.

Case $t<0$ :

$$
\begin{aligned}
\int_{\mathbf{R}}\left[\sup _{w \in U}\left(\left|f_{v}^{\prime}(t, w, x)\right|^{\alpha(w)}\right)\right]^{1+\delta} r(x)^{\delta} \mathrm{d} x \leq & \sum_{j=[t-\epsilon]}^{-1} 2^{(-j+1) \delta} \int_{j}^{j+1} \mathrm{e}^{-\lambda(1+\delta) c(x-t)} \mathrm{d} x \\
& +\sum_{j=0}^{+\infty} 2^{(j+1) \delta} \int_{j}^{j+1} \mathrm{e}^{-\lambda(1+\delta) c(x-t)} \mathrm{d} x \\
\leq & \frac{2^{\delta} \mathrm{e}^{\lambda(1+\delta) c t}\left(\mathrm{e}^{-\lambda(1+\delta) c}-1\right)}{\lambda c(1+\delta)} \sum_{j=[t-\epsilon]}^{-1} 2^{-j \delta} \mathrm{e}^{-\lambda(1+\delta) c j} \\
& +\frac{2^{\delta} \mathrm{e}^{\lambda(1+\delta) c t}\left(\mathrm{e}^{-\lambda(1+\delta) c}-1\right)}{\lambda c(1+\delta)} \sum_{j=0}^{+\infty} 2^{j \delta} \mathrm{e}^{-\lambda(1+\delta) c j} \\
\leq & \frac{2^{\delta} \mathrm{e}^{\lambda(1+\delta) c t}\left(\mathrm{e}^{-\lambda(1+\delta) c}-1\right)}{\lambda c(1+\delta)} \sum_{j=1}^{-[t-\epsilon]} \mathrm{e}^{j(\delta \ln 2+\lambda(1+\delta) c)} \\
& +\frac{2^{\delta} \mathrm{e}^{\lambda(1+\delta) c t}\left(\mathrm{e}^{-\lambda(1+\delta) c}-1\right)}{\lambda c(1+\delta)} \sum_{j=0}^{+\infty} \mathrm{e}^{j(\delta(\ln 2-\lambda c)-\lambda c) .}
\end{aligned}
$$

If $\ln 2-\lambda c<0$, there are no constraints on $\delta$. Otherwise, chose $\delta \in\left(0, \frac{\lambda c}{\ln 2-\lambda c}\right)$. 
Case $t>0$ :

$$
\begin{aligned}
\int_{\mathbf{R}}\left[\sup _{w \in U}\left(\left|f_{v}^{\prime}(t, w, x)\right|^{\alpha(w)}\right)\right]^{1+\delta} r(x)^{\delta} \mathrm{d} x & \leq \sum_{j=[t-\epsilon]}^{+\infty} 2^{(j+1) \delta} \int_{j}^{j+1} \mathrm{e}^{-\lambda(1+\delta) c(x-t)} \mathrm{d} x \\
& \leq \frac{2^{\delta} \mathrm{e}^{\lambda(1+\delta) c t}\left(\mathrm{e}^{-\lambda(1+\delta) c}-1\right)}{\lambda c(1+\delta)} \sum_{j=[t-\epsilon]}^{+\infty} 2^{j \delta} \mathrm{e}^{-\lambda(1+\delta) c j} \\
& \leq \frac{2^{\delta} \mathrm{e}^{\lambda(1+\delta) c t}\left(\mathrm{e}^{-\lambda(1+\delta) c}-1\right)}{\lambda c(1+\delta)} \sum_{j=0}^{+\infty} \mathrm{e}^{j(\delta(\ln 2-\lambda c)-\lambda c)}
\end{aligned}
$$

- (M2) Obvious.

- (M3)

$$
\left[\sup _{w \in U}|\log (r(x))|^{\alpha(w)}\right]^{1+\delta} r(x)^{\delta} \leq|\log 2|^{d(1+\delta)} \sum_{j=1}^{+\infty}|j+1|^{d(1+\delta)} 2^{(j+1) \delta} \mathbf{1}_{[-j,-j+1[\cup[j-1, j[}(x) .
$$

The remaining of the proof is the same as in (M1).

- (M4) $f(v, u, x) \leq 1$.

- (M5) Obvious.

- $(\mathrm{M} 6)$

- (M7)

$$
\int_{\mathbf{R}}|f(v, u, x)|^{2} \mathrm{~d} x=\frac{1}{2 \lambda} .
$$

- (H1) We have $h(u)=\frac{1}{\alpha(u)}$.

$$
\int_{\mathbf{R}}|f(v, v, x)|^{2} \mathrm{~d} x=\frac{1}{2 \lambda}>0 .
$$

- (H2)

$$
\begin{aligned}
\frac{1}{r} \int_{\mathbf{R}}|f(t+r, t, x)-f(t, t, x)|^{\alpha(t)} \mathrm{d} x & =\frac{1}{r} \int_{t}^{t+r} \mathrm{e}^{-\alpha(t) \lambda(x-t)} \mathrm{d} x+\frac{1}{r}\left|\mathrm{e}^{\lambda(t+r)}-\mathrm{e}^{\lambda t}\right|^{\alpha(t)} \int_{t+r}^{+\infty} \mathrm{e}^{-\lambda \alpha(t) x} \mathrm{~d} x \\
& \leq \frac{1}{\lambda \alpha(t)}\left[\frac{\left|\mathrm{e}^{\lambda r}-1\right|^{\alpha(t)}}{r}+\frac{\left(1-\mathrm{e}^{-\lambda \alpha(t) r}\right)}{r}\right]
\end{aligned}
$$

and $\alpha(t) \geq 1$.

- (H3)

$$
\frac{1}{|v-u|} \int_{\mathbf{R}}|f(v, u, x)-f(u, u, x)|^{p} \mathrm{~d} x \leq \frac{1}{\lambda p}\left[\frac{\left|\mathrm{e}^{\lambda|v-u|}-1\right|^{p}}{|v-u|}+\frac{\left(1-\mathrm{e}^{-\lambda p|v-u|}\right)}{|v-u|}\right] .
$$

- $(\mathrm{H} 4)$ Let $g(t) \equiv 1$.

$$
\left|\frac{1}{r} \int_{\mathbf{R}}(f(t+r, t, x)-f(t, t, x))^{2} \mathrm{~d} x-g(t)\right| \leq\left|\frac{1}{2 \lambda} \frac{\left(1-\mathrm{e}^{-2 \lambda r}\right)}{r}-1\right|+\left|\frac{1}{2 \lambda} \frac{\left|\mathrm{e}^{\lambda r}-1\right|^{2}}{r}\right| .
$$

- (H5) Obvious. 


\section{Proof of Theorem 3.7}

Recall the definition of the Lévy multistable field on $[0,1]$ :

$$
X(v, u)=C_{\alpha(u)}^{1 / \alpha(u)} \sum_{i=1}^{\infty} \gamma_{i} \Gamma_{i}^{-1 / \alpha(u)} \mathbf{1}_{[0, v]}\left(V_{i}\right) .
$$

To prove Theorem 3.7, we need a series of lemma:

Lemma 6.16. Assume $\alpha$ is $\mathcal{C}^{1}$. Then, for all $u \in(0,1)$, almost surely,

$$
\sup _{v \in[0,1]} \frac{|X(v, v)-X(v, u)|}{|v-u|}<+\infty .
$$

Proof. in the case of the Lévy multistable field, (5.10) reads:

$$
X(v, v)-X(v, u)=(v-u)\left(\sum_{i=1}^{+\infty} Z_{i}^{1}(v)+\sum_{i=1}^{+\infty} Z_{i}^{3}(v)+\sum_{i=1}^{+\infty} Y_{i}^{1}(v)+\sum_{i=1}^{+\infty} Y_{i}^{3}(v)\right),
$$

where $Z_{i}^{1}, \ldots$ are defined as above. Let $A>0$ and $B>0$ be constants such that $\forall w \in U$, $\left|a^{\prime}(w)\right| \leq A$ and $\left|a(w) \frac{\alpha^{\prime}(w)}{\alpha^{2}(w)}\right| \leq B$. We write $\sum_{i=1}^{+\infty} Z_{i}^{1}(v)=\sum_{j=1}^{+\infty}\left(\sum_{i=2^{j}}^{2^{j+1}-1} Z_{i}^{1}(v)\right)=: \sum_{j=1}^{+\infty} X_{j}^{1}(v)$ and $\sum_{i=1}^{+\infty} Z_{i}^{3}(v)=\sum_{j=1}^{+\infty}\left(\sum_{i=2^{j}}^{2^{j+1}-1} Z_{i}^{3}(v)\right)=: \sum_{j=1}^{+\infty} X_{j}^{3}(v)$. We consider $\left.\liminf \sup _{j}\left|X_{j}^{1}(v)\right| \leq \frac{A j \sqrt{2^{j}}}{2^{j / d}}\right\}$ and $\liminf _{j}\left\{\sup _{v \in[0,1]}\left|X_{j}^{3}(v)\right| \leq \frac{\log (2) B j(j+1) \sqrt{2^{j}}}{2^{j / d}}\right\}$. Let $V^{(1)}, V^{(2)}, \ldots, V^{\left(2^{j}\right)}$ denote the order statistics of the $V_{i}$ (i.e. $\left.V^{(1)}=\min V_{i}, \ldots\right)$. Then:

$$
\begin{aligned}
& \left\{\sup _{v \in[0,1]}\left|X_{j}^{1}(v)\right|>\frac{A j \sqrt{2^{j}}}{2^{j / d}}\right\} \subset \cup_{N \geq 1}^{2^{j}} \cup_{l_{1}, \ldots, l_{N} \in \llbracket 2^{j}, 2^{j+1}-1 \rrbracket}\left(\left\{\left|\sum_{i=1}^{N} \gamma_{l_{i}} a^{\prime}\left(w_{l_{i}}\right) l_{i}^{-1 / \alpha\left(w_{l_{i}}\right)}\right|>\frac{A j \sqrt{2^{j}}}{2^{j / d}}\right\} \ldots\right. \\
& \left.\ldots \cap\left\{V^{(1)}=V_{l_{1}}, V^{(2)}=V_{l_{2}}, \ldots, V^{(N)}=V_{l_{N}}\right\}\right) . \\
& \mathrm{P}\left(\sup _{v \in[0,1]}\left|X_{j}^{1}(v)\right|>\frac{A j \sqrt{2^{j}}}{2^{j / d}}\right) \leq \sum_{N=1}^{2^{j}} \sum_{l_{1}, \ldots, l_{N} \in \llbracket 2^{j}, 2^{j+1}-1 \rrbracket} \frac{\left(2^{j}-N\right) !}{\left(2^{j}\right) !} \mathrm{P}\left(\left|\sum_{i=1}^{N} \gamma_{l_{i}} a^{\prime}\left(w_{l_{i}}\right) l_{i}^{-1 / \alpha\left(w_{l_{i}}\right)}\right|>\frac{A j \sqrt{2^{j}}}{2^{j / d}}\right) \\
& \leq \sum_{N=1}^{2^{j}} \sum_{l_{1}, \ldots, l_{N} \in \llbracket 2^{j}, 2^{j+1}-1 \rrbracket} \frac{\left(2^{j}-N\right) !}{\left(2^{j}\right) !} \mathrm{P}\left(\left|\sum_{i=1}^{N} \gamma_{l_{i}} \frac{a^{\prime}\left(w_{l_{i}}\right)}{A} \frac{2^{j / d}}{l_{i}^{1 / \alpha\left(w_{l_{i}}\right)}}\right|>j \sqrt{N}\right) \\
& \leq \sum_{N=1}^{2^{j}} \sum_{l_{1}, \ldots, l_{N} \in \llbracket 2^{j}, 2^{j+1}-1 \rrbracket} \frac{\left(2^{j}-N\right) !}{\left(2^{j}\right) !} 2 \mathrm{e}^{-\frac{j^{2}}{2}} \\
& \leq 2 \mathrm{e}^{-\frac{j^{2}}{2}} \sum_{N=1}^{2^{j}} \frac{1}{N !} \\
& \leq 2 \mathrm{e}^{1-\frac{j^{2}}{2}}
\end{aligned}
$$


where we have used the following inequality (Lem. 1.5, Chap. 1 in [16]):

$$
\mathrm{P}\left(\left|\sum_{i=1}^{n} u_{i}\right| \geq \lambda \sqrt{n}\right) \leq 2 \mathrm{e}^{-\frac{\lambda^{2}}{2}}
$$

for $\left(u_{i}\right)_{i}$ independent centered random variables verifying $-1 \leq u_{i} \leq 1$, with $u_{i}=\gamma_{l_{i}} \frac{a^{\prime}\left(w_{l_{i}}\right)}{A} \frac{2^{j / d}}{l_{i}^{1 / \alpha\left(w_{l_{i}}\right)}}$ and $\lambda=j$.

We deduce that $\left.\mathrm{P}\left(\liminf \operatorname{sip}_{j \in[0,1]}\left|X_{j}^{1}(v)\right| \leq \frac{A j \sqrt{2^{j}}}{2^{j / d}}\right\}\right)=1$.

Similarly:

$$
\mathrm{P}\left(\sup _{v \in[0,1]}\left|X_{j}^{3}(v)\right|>\frac{\log (2) B j(j+1) \sqrt{2^{j}}}{2^{j / d}}\right) \leq 2 \mathrm{e}^{1-\frac{j^{2}}{2}}
$$

and $\left.\mathrm{P}\left(\liminf \operatorname{sip}_{j}\left|\sup _{v \in[0,1]}^{3}(v)\right| \leq \frac{\log (2) B j(j+1) \sqrt{2^{j}}}{2^{j / d}}\right\}\right)=1$. We work on the event

$$
\liminf _{j}\left\{\sup _{v \in[0,1]}\left|X_{j}^{1}(v)\right| \leq \frac{A j \sqrt{2^{j}}}{2^{j / d}}\right\} \cap \liminf _{j}\left\{\sup _{v \in[0,1]}\left|X_{j}^{3}(v)\right| \leq \frac{\log (2) B j(j+1) \sqrt{2^{j}}}{2^{j / d}}\right\} \cap \liminf _{i}\left\{\Gamma_{i}>1\right\} .
$$

There exists $J_{0} \in \mathbb{N}$ such that $\forall j \geq J_{0}, \sup _{v \in[0,1]}\left|X_{j}^{1}(v)\right| \leq \frac{A j \sqrt{2^{j}}}{2^{j / d}}$ and $\sup _{v \in[0,1]}\left|X_{j}^{3}(v)\right| \leq \frac{\log (2) B j(j+1) \sqrt{2^{j}}}{2^{j / d}}$.

$$
\left|\sum_{i=1}^{+\infty} Z_{i}^{1}(v)\right| \leq \sum_{j=0}^{2^{J_{0}}-1} \frac{A}{i^{1 / d}}+\sum_{j=J_{0}}^{+\infty} A \frac{j}{2^{j\left(\frac{1}{d}-\frac{1}{2}\right)}}
$$

and

$$
\left|\sum_{i=1}^{+\infty} Z_{i}^{3}(v)\right| \leq \sum_{j=0}^{2^{J_{0}}-1} \frac{B \log (i)}{i^{1 / d}}+\sum_{j=J_{0}}^{+\infty} B \log (2) \frac{j(j+1)}{2^{j\left(\frac{1}{d}-\frac{1}{2}\right)}}
$$

thus $\sup _{v \in[0,1]}\left|\sum_{i=1}^{+\infty} Z_{i}^{1}(v)\right|<+\infty$ and $\sup _{v \in[0,1]}\left|\sum_{i=1}^{+\infty} Z_{i}^{3}(v)\right|<+\infty$. 
Fix $i_{0} \in \mathbb{N}$ such that $\forall i \geq i_{0}, \Gamma_{i}>1$.

$$
\left|\sum_{i=1}^{i_{0}} Y_{i}^{1}(v)\right| \leq A \sum_{i=1}^{i_{0}}\left(\frac{1}{\Gamma_{i}^{1 / c}}+\frac{1}{i^{1 / d}}\right)
$$

and

$$
\begin{aligned}
& \left|\sum_{i=1}^{i_{0}} Y_{i}^{3}(v)\right| \leq B \sum_{i=1}^{i_{0}}\left(\left|\frac{\log \Gamma_{i}}{\Gamma_{i}^{1 / c}}\right|+\frac{\log (i)}{i^{1 / d}}\right) \text {. } \\
& \left|\sum_{i=i_{0}}^{+\infty} Y_{i}^{1}(v)\right| \leq A \sum_{i=i_{0}}^{+\infty}\left|\Gamma_{i}^{-1 / \alpha\left(x_{i}\right)}-i^{-1 / \alpha\left(x_{i}\right)}\right| \mathbf{1}_{\left\{1<\Gamma_{i} \leq \frac{i}{2}\right\}} \\
& +A \sum_{i=i_{0}}^{+\infty}\left|\Gamma_{i}^{-1 / \alpha\left(x_{i}\right)}-i^{-1 / \alpha\left(x_{i}\right)}\right| \mathbf{1}_{\left\{\frac{i}{2}<\Gamma_{i} \leq 2 i\right\}} \\
& +A \sum_{i=i_{0}}^{+\infty}\left|\Gamma_{i}^{-1 / \alpha\left(x_{i}\right)}-i^{-1 / \alpha\left(x_{i}\right)}\right| \mathbf{1}_{\left\{\Gamma_{i}>2 i\right\}} \\
& \left|\sum_{i=i_{0}}^{+\infty} Y_{i}^{1}(v)\right| \leq 2 A \sum_{i=i_{0}}^{+\infty}\left(\mathbf{1}_{\left\{1<\Gamma_{i} \leq \frac{i}{2}\right\}}+\mathbf{1}_{\left\{\Gamma_{i}>2 i\right\}}\right)+A \sum_{i=i_{0}}^{+\infty}\left|\Gamma_{i}^{-1 / \alpha\left(x_{i}\right)}-i^{-1 / \alpha\left(x_{i}\right)}\right| \mathbf{1}_{\left\{\frac{i}{2}<\Gamma_{i} \leq 2 i\right\}} \\
& \leq 2 A \sum_{i=i_{0}}^{+\infty}\left(\mathbf{1}_{\left\{1<\Gamma_{i} \leq \frac{i}{2}\right\}}+\mathbf{1}_{\left\{\Gamma_{i}>2 i\right\}}\right)+K_{c, d} \sum_{i=i_{0}}^{+\infty} \frac{1}{i^{\frac{1}{d}}}\left|\frac{\Gamma_{i}}{i}-1\right| \text {. } \\
& \left|\sum_{i=i_{0}}^{+\infty} Y_{i}^{3}(v)\right| \leq B \sum_{i=i_{0}}^{+\infty}\left|\log \left(\Gamma_{i}\right) \Gamma_{i}^{-1 / \alpha\left(x_{i}\right)}-\log (i) i^{-1 / \alpha\left(x_{i}\right)}\right| \mathbf{1}_{\left\{1<\Gamma_{i} \leq \frac{i}{2}\right\}} \\
& +B \sum_{i=i_{0}}^{+\infty}\left|\log \left(\Gamma_{i}\right) \Gamma_{i}^{-1 / \alpha\left(x_{i}\right)}-\log (i) i^{-1 / \alpha\left(x_{i}\right)}\right| \mathbf{1}_{\left\{\frac{i}{2}<\Gamma_{i} \leq 2 i\right\}} \\
& +B \sum_{i=i_{0}}^{+\infty}\left|\log \left(\Gamma_{i}\right) \Gamma_{i}^{-1 / \alpha\left(x_{i}\right)}-\log (i) i^{-1 / \alpha\left(x_{i}\right)}\right| \mathbf{1}_{\left\{\Gamma_{i}>2 i\right\}}, \\
& \left|\sum_{i=i_{0}}^{+\infty} Y_{i}^{3}(v)\right| \leq K \sum_{i=i_{0}}^{+\infty} \log (i)\left(\mathbf{1}_{\left\{1<\Gamma_{i} \leq \frac{i}{2}\right\}}+\mathbf{1}_{\left\{\Gamma_{i}>2 i\right\}}\right) \\
& +B \sum_{i=i_{0}}^{+\infty}\left|\log \left(\Gamma_{i}\right) \Gamma_{i}^{-1 / \alpha\left(x_{i}\right)}-\log (i) i^{-1 / \alpha\left(x_{i}\right)}\right| \mathbf{1}_{\left\{\frac{i}{2}<\Gamma_{i} \leq 2 i\right\}} \\
& \leq K \sum_{i=i_{0}}^{+\infty} \log (i)\left(\mathbf{1}_{\left\{1<\Gamma_{i} \leq \frac{i}{2}\right\}}+\mathbf{1}_{\left\{\Gamma_{i}>2 i\right\}}\right)+K_{c, d} \sum_{i=i_{0}}^{+\infty} \frac{\log (i)}{i^{\frac{1}{d}}}\left|\frac{\Gamma_{i}}{i}-1\right| .
\end{aligned}
$$

Finally, $\sup _{v \in[0,1]}\left|\sum_{i=1}^{+\infty} Y_{i}^{1}(v)\right|<+\infty$ and $\sup _{v \in[0,1]}\left|\sum_{i=1}^{+\infty} Y_{i}^{3}(v)\right|<+\infty$.

As a consequence, $\sup _{v \in[0,1]} \frac{|X(v, v)-X(v, u)|}{|v-u|}<+\infty$ 
Lemma 6.17. For all $u \in(0,1)$ and all $\eta \in\left(0, \frac{1}{\alpha(u)}\right)$, one has, almost surely,

$$
\sup _{v \in[0,1]}\left|\frac{X(v, u)-X(u, u)}{|v-u|^{\eta}}\right|<+\infty .
$$

Proof. Let $\eta \in\left(0, \frac{1}{\alpha(u)}\right), m \in \mathbb{N}, C_{j}=\cap_{i=2^{j}}^{2^{j+1}-1}\left\{V_{i} \notin\left[u-\frac{1}{j^{2} 2^{j}}, u+\frac{1}{j^{2} 2^{j}}\right]\right\}$,

$$
D_{j}^{m}=\left\{\sup _{\frac{1}{2^{m+1}} \leq|v-u| \leq \frac{1}{2^{m}}}\left|\sum_{i=2^{j}}^{2^{j+1}-1} \gamma_{i} i^{-1 / \alpha(u)} \frac{\mathbf{1}_{[u, v]}\left(V_{i}\right)}{|v-u|^{\eta}}\right| \leq \frac{1}{j^{2}}\right\},
$$

and $D_{j}=\cap_{m \geq 0} D_{j}^{m} . D_{j}$ may be written:

$$
D_{j}=\left\{\sup _{v \in[0,1]}\left|\sum_{i=2^{j}}^{2^{j+1}-1} \gamma_{i} i^{-1 / \alpha(u)} \frac{\mathbf{1}_{[u, v]}\left(V_{i}\right)}{|v-u|^{\eta}}\right| \leq \frac{1}{j^{2}}\right\} .
$$

Let us evaluate $\liminf C_{j}$.

$$
\mathrm{P}\left(\overline{C_{j}}\right) \leq \sum_{i=2^{j}}^{2^{j+1}-1} \frac{1}{j^{2} 2^{j}}=\frac{1}{j^{2}}
$$

and thus $\mathrm{P}\left(\liminf _{j} C_{j}\right)=1$. Now,

$$
\begin{aligned}
\mathrm{P}\left(\overline{D_{j}}\right) & \leq \frac{1}{j^{2}}+\mathrm{P}\left(\overline{D_{j}} \cap C_{j}\right) \\
& =\frac{1}{j^{2}}+\mathrm{P}\left(\cup_{m \geq 0}\left(\overline{D_{j}^{m}} \cap C_{j}\right)\right) \\
& \leq \frac{1}{j^{2}}+\sum_{m=0}^{+\infty} \mathrm{P}\left(\overline{D_{j}^{m}} \cap C_{j}\right) .
\end{aligned}
$$

We consider several cases, depending on the respective values of $j$ and $m$ :

- If $m>j+\frac{2}{\log (2)} \log j$,

$$
\mathrm{P}\left(\overline{D_{j}^{m}} \cap C_{j}\right)=0
$$

- If $j+\frac{2}{\log (2)} \log j \geq m \geq j$,

$$
\mathrm{P}\left(\overline{D_{j}^{m}}\right) \leq \mathrm{P}\left(\sup _{\frac{1}{2^{m+1}} \leq|v-u| \leq \frac{1}{2^{m}}}\left|\sum_{i=2^{j}}^{2^{j+1}-1} \gamma_{i} i^{-1 / \alpha(u)} \mathbf{1}_{[u, v]}\left(V_{i}\right)\right| \geq \frac{1}{2^{(m+1) \eta} j^{2}}\right) .
$$

Let $J_{0} \in \mathbb{N}$ be such that for all $j>J_{0}, 2^{j\left(\frac{1}{\alpha(u)}-\eta\right)}>2^{\eta} j^{3+\frac{2 \eta}{\log (2)}}$. The event:

$$
\left\{\sup _{\frac{1}{2^{m+1}} \leq|v-u| \leq \frac{1}{2^{m}}}\left|\sum_{i=2^{j}}^{2^{j+1}-1} \gamma_{i} i^{-1 / \alpha(u)} \mathbf{1}_{[u, v]}\left(V_{i}\right)\right| \geq \frac{1}{2^{(m+1) \eta} j^{2}}\right\}
$$


is included in the event

$$
\begin{array}{r}
\cup_{N \geq 1}^{2^{j}}\left(\cup_{l_{1}, \ldots, l_{N} \in \llbracket 2^{j}, 2^{j+1}-1 \rrbracket}\left\{\left|\sum_{i=1}^{N} \gamma_{l_{i}} l_{i}^{-1 / \alpha(u)}\right|>\frac{1}{2^{(m+1) \eta} j^{2}}\right\} \cap\left(\cap_{i=1}^{N}\left\{\left|V_{l_{i}}-u\right| \in\left[\frac{1}{2^{m+1}}, \frac{1}{2^{m}}\right]\right\}\right) \ldots\right. \\
\left.\ldots \cap\left(\cap_{k \neq l_{i}}\left\{\left|V_{k}-u\right| \notin\left[\frac{1}{2^{m+1}}, \frac{1}{2^{m}}\right]\right\}\right)\right) .
\end{array}
$$

Notice that for $j \geq J_{0}$ and $N<j, \mathrm{P}\left(\left|\sum_{i=1}^{N} \gamma_{l_{i}} l_{i}^{-1 / \alpha(u)}\right|>\frac{1}{2^{(m+1) \eta} j^{2}}\right)=0$, and thus

$$
\begin{aligned}
& \mathrm{P}\left(\overline{D_{j}^{m}}\right) \leq \sum_{N=j}^{2^{j}} \sum_{l_{1}, \ldots, l_{N} \in \llbracket 2^{j}, 2^{j+1}-1 \rrbracket} \mathrm{P}\left(\left|\sum_{i=1}^{N} \gamma_{l_{i}} l_{i}^{-1 / \alpha(u)}\right|>\frac{1}{2^{(m+1) \eta} j^{2}}\right) \mathrm{P}\left(\cap_{i=1}^{N}\left\{\left|V_{l_{i}}-u\right| \in\left[\frac{1}{2^{m+1}}, \frac{1}{2^{m}}\right]\right\}\right) \\
& \leq \sum_{N=j}^{2^{j}} \frac{1}{2^{(m+1) N}} \sum_{l_{1}, \ldots, l_{N} \in \llbracket 2^{j}, 2^{j+1}-1 \rrbracket} \mathrm{P}\left(\left|\sum_{i=1}^{N} \gamma_{l_{i}} l_{i}^{-1 / \alpha(u)}\right|>\frac{1}{2^{(m+1) \eta} j^{2}}\right) \\
& \leq \sum_{N=j}^{2^{j}} \frac{1}{2^{(m+1) N}} \sum_{l_{1}, \ldots, l_{N} \in \llbracket 2^{j}, 2^{j+1}-1 \rrbracket} j^{4} 2^{2(m+1) \eta} \sum_{i=2^{j}}^{2^{j+1}-1} \frac{1}{i^{\frac{2}{\alpha(u)}}} \\
& \leq \sum_{N=j}^{2^{j}} \frac{j^{4} 2^{2(m+1) \eta}}{2^{(m+1) N}} 2^{j\left(1-\frac{2}{\alpha(u)}\right)} C_{2^{j}}^{N} \\
& \leq j^{4} 2^{2\left(j+\frac{2}{\log (2)} \log j+1\right) \eta-j \frac{2}{\alpha(u)}} \sum_{N=j}^{2^{j}} \frac{2^{j} C_{2^{j}}^{N}}{2^{(m+1) N}} \\
& \leq j^{\left.4+\frac{4 \eta}{\log (2)} 2^{2 j\left(\eta-\frac{1}{\alpha(u)}\right.}\right)} \sum_{N=j}^{2^{j}} \frac{2^{j-N} 2^{(j-m) N}}{N !} \\
& \leq 3 j^{4+\frac{4 \eta}{\log (2)}} 2^{2 j\left(\eta-\frac{1}{\alpha(u)}\right)} \text {. }
\end{aligned}
$$

- When $j \geq m \geq \frac{\log (j)}{\log (2)}$, the same computations lead to:

$$
\begin{aligned}
\sum_{N=j 2^{j-m}}^{2^{j}} \sum_{l_{1}, \ldots, l_{N} \in \llbracket 2^{j}, 2^{j+1}-1 \rrbracket} \mathrm{P}\left(\left|\sum_{i=1}^{N} \gamma_{l_{i}} l_{i}^{-1 / \alpha(u)}\right|>\frac{1}{2^{(m+1) \eta} j^{2}}\right) \mathrm{P}\left(\cap_{i=1}^{N}\left\{\left|V_{l_{i}}-u\right| \in\left[\frac{1}{2^{m+1}}, \frac{1}{2^{m}}\right]\right\}\right) \\
\leq \sum_{N=j 2^{j-m}} \frac{j^{4} 2^{2(m+1) \eta}}{2^{(m+1) N}} 2^{j\left(1-\frac{2}{\alpha(u)}\right)} C_{2^{j}}^{N} \\
\leq j^{4} 2^{2(m+1) \eta-2 j / \alpha(u)} \sum_{N=j 2^{j-m}}^{2^{j}} \frac{2^{j-N} 2^{(j-m) N}}{N !} \\
\left.\leq j^{4} 2^{2 \eta} 2^{2 j\left(\eta-\frac{1}{\alpha(u)}\right.}\right) \sum_{N=j 2^{j-m}}^{+\infty} \frac{2^{(j-m) N}}{N !} \\
\leq K j^{4} 2^{2 j\left(\eta-\frac{1}{\alpha(u)}\right)} \frac{\mathrm{e}^{2^{j-m}} 2^{(j-m)\left(j 2^{j-m}+1\right)}}{\left(j 2^{j-m}+1\right) !}
\end{aligned}
$$


where we have used the estimate $\sum_{n \geq N} \frac{x^{n}}{n !} \leq \mathrm{e}^{x} \frac{x^{N+1}}{(N+1) !}$. We arrive at:

$$
\begin{aligned}
\mathrm{P}\left(\overline{D_{j}^{m}}\right) \leq & K j^{4} 2^{2 j\left(\eta-\frac{1}{\alpha(u)}\right)} \\
& +\sum_{N=1}^{j 2^{j-m}} \frac{1}{2^{(m+1) N}}\left(1-\frac{1}{2^{m+1}}\right)^{2^{j}-N} \sum_{l_{1}, \ldots, l_{N} \in \llbracket 2^{j}, 2^{j+1}-1 \rrbracket} \mathrm{P}\left(\left|\sum_{i=1}^{N} \gamma_{l_{i}} l_{i}^{-1 / \alpha(u)}\right|>\frac{1}{2^{(m+1) \eta} j^{2}}\right) .
\end{aligned}
$$

We need to distinguish two cases depending on the value of $\eta$. If $\eta \leq \frac{1}{2}$, fix $J_{1} \in \mathbb{N}$ such that for all $j \geq J_{1}$, $2^{j\left(\frac{1}{\alpha(u)}-\frac{1}{2}\right)}>2^{1 / \alpha(u)} j^{3} \sqrt{j}$. If $\eta>\frac{1}{2}$, fix $J_{1} \in \mathbb{N}$ such that for all $j \geq J_{1}, 2^{j\left(\frac{1}{\alpha(u)}-\eta\right)}>2^{1 / \alpha(u)} j^{3} \sqrt{j}$. Then for all $\eta$ and all $j \geq J_{1}$, one has $\frac{2^{j / \alpha(u)}}{j^{3} \sqrt{2^{j-m}} 2^{(m+1) \eta}} \geq 1$ and

$$
\begin{aligned}
\mathrm{P}\left(\left|\sum_{i=1}^{N} \gamma_{l_{i}} l_{i}^{-1 / \alpha(u)}\right|>\frac{1}{2^{(m+1) \eta} j^{2}}\right) & \leq \mathrm{P}\left(\left|\sum_{i=1}^{N} \gamma_{l_{i}}\left(\frac{2^{j}}{l_{i}}\right)^{1 / \alpha(u)}\right|>j \sqrt{N}\right) \\
& \leq 2 \mathrm{e}^{-j^{2} / 2} .
\end{aligned}
$$

We then get

$$
\begin{aligned}
\mathrm{P}\left(\overline{D_{j}^{m}}\right) & \leq K j^{4} 2^{2 j\left(\eta-\frac{1}{\alpha(u)}\right)}+\sum_{N=1}^{j 2^{j-m}} \frac{1}{2^{(m+1) N}}\left(1-\frac{1}{2^{m+1}}\right)^{2^{j}-N} C_{2^{j}}^{N} 2 \mathrm{e}^{-j^{2} / 2} \\
& \leq K j^{4} 2^{2 j\left(\eta-\frac{1}{\alpha(u)}\right)}+2 \mathrm{e}^{-j^{2} / 2} \sum_{N=1}^{2^{j}} \frac{1}{2^{(m+1) N}}\left(1-\frac{1}{2^{m+1}}\right)^{2^{j}-N} C_{2^{j}}^{N} \\
& \leq K j^{4} 2^{2 j\left(\eta-\frac{1}{\alpha(u)}\right)}+2 \mathrm{e}^{-j^{2} / 2} .
\end{aligned}
$$

- Assume finally that $m \leq \frac{\log (j)}{\log (2)}$.

Fix $J_{2} \in \mathbb{N}$ such that for all $j \geq J_{2}, 2^{j\left(\frac{1}{\alpha(u)}-\frac{1}{2}\right)}>2^{1 / \alpha(u)} j^{3+\eta}$. Then, for $j \geq J_{2}$, one has $\frac{2^{j / \alpha(u)}}{j^{3} \sqrt{2^{j}} 2^{(m+1) \eta}} \geq 1$ and computations similar the ones above lead to

$$
\begin{aligned}
\mathrm{P}\left(\overline{D_{j}^{m}}\right) & \leq \sum_{N=1}^{2^{j}} \frac{1}{2^{(m+1) N}}\left(1-\frac{1}{2^{m+1}}\right)^{2^{j}-N} \sum_{l_{1}, \ldots, l_{N} \in \llbracket 2^{j}, 2^{j+1}-1 \rrbracket} \mathrm{P}\left(\left|\sum_{i=1}^{N} \gamma_{l_{i}} l_{i}^{-1 / \alpha(u)}\right|>\frac{1}{2^{(m+1) \eta} j^{2}}\right) \\
& \leq \sum_{N=1}^{2^{j}} \frac{1}{2^{(m+1) N}}\left(1-\frac{1}{2^{m+1}}\right)^{2^{j}-N} \sum_{l_{1}, \ldots, l_{N} \in \llbracket 2^{j}, 2^{j+1}-1 \rrbracket} \mathrm{P}\left(\left|\sum_{i=1}^{N} \gamma_{l_{i}}\left(\frac{2^{j / \alpha(u)}}{l_{i}}\right)^{1 / \alpha(u)}\right|>j \sqrt{N}\right) \\
& \leq 2 \mathrm{e}^{-j^{2} / 2} .
\end{aligned}
$$

We thus get that, for $j \geq \max \left(J_{0}, J_{1}, J_{2}\right)$,

$$
\sum_{m=0}^{+\infty} \mathrm{P}\left(\overline{D_{j}^{m}} \cap C_{j}\right) \leq K \log (j) j^{4+\frac{4 \eta}{\log (2)}} 2^{2 j\left(\eta-\frac{1}{\alpha(u)}\right)},
$$

and thus $\mathrm{P}\left(\liminf { }_{j} D_{j}\right)=1$. 
On the event $\liminf _{j} C_{j} \cap \liminf _{j} D_{j}$, we may fix $j_{0} \in \mathbb{N}$ such that for all $j \geq j_{0}$,

$$
\sup _{v \in[0,1]}\left|\sum_{i=2^{j}}^{2^{j+1}-1} \gamma_{i} i^{-1 / \alpha(u)} \frac{\mathbf{1}_{[u, v]}\left(V_{i}\right)}{|v-u|^{\eta}}\right| \leq \frac{1}{j^{2}} .
$$

Since $\sup _{v \in[0,1]}\left|\sum_{i=1}^{2^{j} 0-1} \gamma_{i} i^{-1 / \alpha(u)} \frac{\mathbf{1}_{[u, v]}\left(V_{i}\right)}{|v-u|^{\eta}}\right|<+\infty$, we obtain

$$
\sup _{v \in[0,1]}\left|\sum_{i=1}^{+\infty} \gamma_{i} i^{-1 / \alpha(u)} \frac{\mathbf{1}_{[u, v]}\left(V_{i}\right)}{|v-u|^{\eta}}\right|<+\infty .
$$

Let us now deal with

$$
\begin{aligned}
& E_{j}=\left\{\sup _{v \in[0,1]}\left|\sum_{i=2^{j}}^{2^{j+1}-1} \gamma_{i}\left(\Gamma_{i}^{-1 / \alpha(u)}-i^{-1 / \alpha(u)}\right) \frac{\mathbf{1}_{[u, v]}\left(V_{i}\right)}{|v-u|^{\eta}}\right| \leq \frac{1}{j^{2}}\right\} \cdot \\
& \mathrm{P}\left(\overline{E_{j}}\right) \leq \frac{1}{j^{2}}+\mathrm{P}\left(\overline{E_{j}} \cap C_{j}\right) \\
& \leq \frac{1}{j^{2}}+\mathrm{P}\left(2^{j \eta} j^{2 \eta} \sup _{v \in[0,1]}\left|\sum_{i=2^{j}}^{2^{j+1}-1} \gamma_{i}\left(\Gamma_{i}^{-1 / \alpha(u)}-i^{-1 / \alpha(u)}\right) \mathbf{1}_{[u, v]}\left(V_{i}\right)\right|>\frac{1}{j^{2}}\right) \\
& \leq \frac{1}{j^{2}}+\mathrm{P}\left(\sum_{i=2^{j}}^{2^{j+1}-1}\left|\left(\Gamma_{i}^{-1 / \alpha(u)}-i^{-1 / \alpha(u)}\right)\right|>\frac{1}{2^{j \eta} j^{2(1+\eta)}}\right) \\
& \leq \frac{1}{j^{2}}+2^{j \eta} j^{2(1+\eta)} \sum_{i=2^{j}}^{2^{j+1}-1} \mathrm{E}\left|\Gamma_{i}^{-1 / \alpha(u)}-i^{-1 / \alpha(u)}\right| \\
& \leq \frac{1}{j^{2}}+2^{j \eta} j^{2(1+\eta)} \sum_{i=2^{j}}^{2^{j+1}-1} 2\left(\mathrm{P}\left(\Gamma_{i}<\frac{i}{2}\right)+\mathrm{P}\left(\Gamma_{i}>2 i\right)\right) \\
&+2^{j \eta} j^{2(1+\eta)} \sum_{i=2^{j}}^{2^{j+1}-1} \mathrm{E}\left|\Gamma_{i}^{-1 / \alpha(u)}-i^{-1 / \alpha(u)}\right| \mathbf{1}_{\left\{\frac{i}{2}<\Gamma_{i}<2 i\right\}} .
\end{aligned}
$$

However

$$
\begin{aligned}
\mathrm{E}\left|\Gamma_{i}^{-1 / \alpha(u)}-i^{-1 / \alpha(u)}\right| \mathbf{1}_{\left\{\frac{i}{2}<\Gamma_{i}<2 i\right\}} & \leq \frac{1}{i^{1 / \alpha(u)}} K_{u} \mathrm{E}\left|\frac{\Gamma_{i}}{i}-1\right| \\
& \leq K_{u} \frac{1}{i^{1+\frac{1}{\alpha(u)}}}
\end{aligned}
$$

and

$$
2^{j \eta} j^{2(1+\eta)} \sum_{i=2^{j}}^{2^{j+1}-1} \mathrm{E}\left|\Gamma_{i}^{-1 / \alpha(u)}-i^{-1 / \alpha(u)}\right| \mathbf{1}_{\left\{\frac{i}{2}<\Gamma_{i}<2 i\right\}} \leq K j^{2(1+\eta)} 2^{j\left(\eta-\frac{1}{\alpha(u)}\right)} .
$$


We thus obtain $\mathrm{P}\left(\liminf _{j} E_{j}\right)=1$. As a consequence, $\sup _{v \in[0,1]}\left|\sum_{i=1}^{+\infty} \gamma_{i}\left(\Gamma_{i}^{-1 / \alpha(u)}-i^{-1 / \alpha(u)}\right) \frac{\mathbf{1}_{[u, v]}\left(V_{i}\right)}{|v-u|^{\eta}}\right|<+\infty$ and finally

$$
\sup _{v \in[0,1]}\left|\frac{X(v, u)-X(u, u)}{|v-u|^{\eta}}\right|<+\infty \text {. }
$$

Lemma 6.18. For all $u \in(0,1)$, one has almost surely, for all $\eta \in\left(0, \frac{1}{\alpha(u)}\right)$,

$$
\sup _{v \in[0,1]} \frac{|X(v, u)-X(u, u)|}{|v-u|^{\eta}}<+\infty .
$$

Proof. Fix $u \in(0,1)$. Lemma 6.17 yields that, for all $\eta \in\left(0, \frac{1}{\alpha(u)}\right)$, we may choose an $\Omega_{\eta}$ having probability one and such that, on $\Omega_{\eta}, \sup _{v \in[0,1]}\left|\frac{X(v, u)-X(u, u)}{|v-u|^{\eta}}\right|<+\infty$. Thus, on $\Omega=\cap_{j \geq 0} \Omega_{\frac{1}{\alpha(u)}-\frac{1}{2^{j}}}$, which still has probability one, it holds that, for all $\eta \in\left(0, \frac{1}{\alpha(u)}\right), \sup _{v \in[0,1]} \frac{|X(v, u)-X(u, u)|}{|v-u|^{\eta}}<+\infty$.

Proof of Theorem 3.7. From Theorem 3.6, we already know that $\mathcal{H}_{u} \leq \frac{1}{\alpha(u)}$. To prove the reverse inequality, we treat separately the situations where $\alpha<1$ and $\alpha \geq 1$.

- Consider first the case $0<\alpha(u)<1$.

Write:

$$
Y(v)-Y(u)=X(v, v)-X(v, u)+X(v, u)-X(u, u)
$$

By Lemma 6.18, we know that the Hölder regularity of $v \mapsto X(v, u)-X(u, u)$ at $u$ is almost surely not smaller than $\frac{1}{\alpha(u)}$. Now, by applying the finite increments theorem to the functions $t \mapsto C_{t}^{1 / t} \Gamma_{i}^{-1 / t}$, we get

$$
\begin{aligned}
X(v, v)-X(v, u) & =\sum_{i=1}^{\infty} \gamma_{i} \mathbf{1}_{[0, v]}\left(V_{i}\right)\left(C_{\alpha(v)}^{1 / \alpha(v)} \Gamma_{i}^{-1 / \alpha(v)}-C_{\alpha(u)}^{1 / \alpha(u)} \Gamma_{i}^{-1 / \alpha(u)}\right) \\
& =(\alpha(v)-\alpha(u)) \sum_{i=1}^{\infty} \gamma_{i} \mathbf{1}_{[0, v]}\left(V_{i}\right)\left(C P\left(\alpha\left(w_{i}\right)\right)-C_{\alpha\left(w_{i}\right)}^{1 / \alpha\left(w_{i}\right)} \frac{\log \Gamma_{i}}{\alpha\left(w_{i}\right)^{2}}\right) \Gamma_{i}^{-1 / \alpha\left(w_{i}\right)}
\end{aligned}
$$

where, for each $i, w_{i} \in[u, v]$ (or $[v, u]$ ), and $C P$ denotes the derivative of the function $t \mapsto C_{t}^{1 / t}$. However,

$$
\begin{aligned}
\left|\sum_{i=1}^{\infty} \gamma_{i} \mathbf{1}_{[0, v]}\left(V_{i}\right)\left(C p\left(\alpha\left(w_{i}\right)\right)-\frac{\log \Gamma_{i}}{\alpha\left(w_{i}\right)^{2}}\right) \Gamma_{i}^{-1 / \alpha\left(w_{i}\right)}\right| & \leq \sum_{i=1}^{\infty}\left|C P\left(\alpha\left(w_{i}\right)\right)-C_{\alpha\left(w_{i}\right)}^{1 / \alpha\left(w_{i}\right)} \frac{\log \Gamma_{i}}{\alpha\left(w_{i}\right)^{2}}\right| \Gamma_{i}^{-1 / \alpha\left(w_{i}\right)} \\
& \leq K \sum_{i=1}^{\infty}\left(1+\left|\log \Gamma_{i}\right|\right)\left(\Gamma_{i}^{-1 / c}+\Gamma_{i}^{-1 / d}\right) .
\end{aligned}
$$

Thus the quantity $T(u, v)=\sum_{i=1}^{\infty} \gamma_{i} \mathbf{1}_{[0, v]}\left(V_{i}\right)\left(C P\left(\alpha\left(w_{i}\right)\right)-C_{\alpha\left(w_{i}\right)}^{1 / \alpha\left(w_{i}\right)} \frac{\log \Gamma_{i}}{\alpha\left(w_{i}\right)^{2}}\right) \Gamma_{i}^{-1 / \alpha\left(w_{i}\right)}$ is, uniformly in $v$, almost surely finite and not 0 . As a consequence, the function $v \mapsto X(v, v)-X(v, u)=(\alpha(u)-\alpha(v)) T(u, v)$ has almost surely the same Hölder exponent at $u$ as the function $v \mapsto \alpha(v)$ at $u$. If $\mathcal{H}_{u}^{\alpha}<\frac{1}{\alpha(u)}$, this entails that $Y$ has exponent $\mathcal{H}_{u}^{\alpha}$ at $u$. If $\mathcal{H}_{u}^{\alpha}>\frac{1}{\alpha(u)}$, then the exponent of $Y$ at $u$ is at least $\frac{1}{\alpha(u)}$ and thus exactly $\frac{1}{\alpha(u)}$ by Theorem 3.6.

- Assume now that $1 \leq \alpha(u)<2$. Let $\eta<\frac{1}{\alpha(u)}$ and $\delta \in\left(\eta, \frac{1}{\alpha(u)}\right)$. Then:

$$
\frac{|Y(v)-Y(u)|}{|v-u|^{\eta}} \leq \frac{|X(v, v)-X(v, u)|}{|v-u|^{\eta}}+\frac{|X(v, u)-X(u, u)|}{|v-u|^{\eta}} .
$$


By Lemma 6.18, there exists $K>0$ such that $\frac{|X(v, u)-X(u, u)|}{|v-u|^{\eta}} \leq K|v-u|^{\delta-\eta}$, and, by Lemma 6.16, there exists $K>0$ such that $\frac{|X(v, v)-X(v, u)|}{|v-u|^{\eta}} \leq K|v-u|^{1-\eta}$. This entails $\lim _{v \rightarrow u} \frac{|Y(v)-Y(u)|}{|v-u|^{\eta}}=0$ and

$$
\mathcal{H}_{u} \geq \frac{1}{\alpha(u)}
$$

\section{Assumptions}

We need to make a series of assumptions on the kernel that define the multistable processes. These asumptions are of three kinds: regularity condition that entail localisability, moment conditions related to the fact that we work in certain functional spaces and finally, Hölder conditions which enable to transfer the behaviour of $f$ to the one of $Y$.

\section{Regularity}

- (R1) The family of functions $v \rightarrow f(t, v, x)$ is differentiable for all $(v, t)$ in $U^{2}$ and almost all $x$ in $E$. The derivatives of $f$ with respect to $v$ are denoted by $f_{v}^{\prime}$.

\section{Moments conditions}

- (M1) There exists $\delta>\frac{d}{c}-1$ such that:

$$
\sup _{t \in U} \int_{\mathbf{R}}\left[\sup _{w \in U}\left(S|f(t, w, x)|^{\alpha(w)}\right)\right]^{1+\delta} r(x)^{\delta} m(\mathrm{~d} x)<\infty .
$$

- (M2) There exists $\delta>\frac{d}{c}-1$ such that:

$$
\sup _{t \in U} \int_{\mathbf{R}}\left[\sup _{w \in U}\left(\left|f_{v}^{\prime}(t, w, x)\right|^{\alpha(w)}\right)\right]^{1+\delta} r(x)^{\delta} m(\mathrm{~d} x)<\infty .
$$

- (M3) There exists $\delta>\frac{d}{c}-1$ such that:

$$
\sup _{t \in U} \int_{\mathbf{R}}\left[\sup _{w \in U}\left[|f(t, w, x) \log (r(x))|^{\alpha(w)}\right]\right]^{1+\delta} r(x)^{\delta} m(\mathrm{~d} x)<\infty .
$$

- (M4) There exists $K_{U}>0$ such that $\forall v \in U, \forall u \in U, \forall x \in \mathbf{R}$,

$$
|f(v, u, x)| \leq K_{U}
$$

- (M5) There exists $K_{U}>0$ such that $\forall v \in U, \forall u \in U, \forall x \in \mathbf{R}$,

$$
\left|f_{v}^{\prime}(v, u, x)\right| \leq K_{U}
$$

- (M6) There exists $K_{U}>0$ such that $\forall v \in U, \forall u \in U$,

$$
\int_{\mathbf{R}}|f(v, u, x)|^{2} m(\mathrm{~d} x) \leq K_{U}
$$

- $(\mathrm{M} 7)$

$$
\inf _{v \in U} \int_{\mathbf{R}} f(v, v, x)^{2} m(\mathrm{~d} x)>0
$$




\section{Hölder conditions}

- (H1) There exists a function $h$ defined on $U$ and $K_{U}>0$ such that $\forall v \in U, \forall u \in U, \forall x \in \mathbf{R}$,

$$
\frac{1}{|v-u|^{h(u)-1 / \alpha(u)}}|f(v, u, x)-f(u, u, x)| \leq K_{U} .
$$

- (H2) There exists a function $h$ defined on $U, \varepsilon_{0}>0$ and $K_{U}>0$ such that $\forall r<\varepsilon_{0}$,

$$
\frac{1}{r^{h(t) \alpha(t)}} \int_{\mathbf{R}}|f(t+r, t, x)-f(t, t, x)|^{\alpha(t)} m(\mathrm{~d} x) \leq K_{U} .
$$

- (H3) There exists a function $h$ defined on $U, p \in(d, 2), p \geq 1$ and $K_{U}>0$ such that $\forall v \in U, \forall u \in U$,

$$
\frac{1}{|v-u|^{1+p\left(h(u)-\frac{1}{\alpha(u)}\right)}} \int_{\mathbf{R}}|f(v, u, x)-f(u, u, x)|^{p} m(\mathrm{~d} x) \leq K_{U} .
$$

- (H4) There exists a function $h$ and a positive function $g$ defined on $U$ such that

$$
\limsup _{r \rightarrow 0}\left|\frac{1}{\operatorname{su}_{t \in U}}\right| \frac{1}{\left.r^{1+2(h(t)-1 / \alpha(t))}\right)} \int_{\mathbf{R}}(f(t+r, t, x)-f(t, t, x))^{2} m(\mathrm{~d} x)-g(t) \mid=0 .
$$

- (H5) $\exists K_{U}>0$ such that, $\forall v \in U, \forall u \in U$,

$$
\frac{1}{|v-u|^{2}} \int_{\mathbf{R}}|f(v, v, x)-f(v, u, x)|^{2} m(\mathrm{~d} x) \leq K_{U}
$$

\section{REFERENCES}

[1] A. Ayache and J. Lévy Véhel, The generalized multifractional Brownian motion. Stat. Inference Stoch. Process. 3 (2000) $7-18$.

[2] A. Benassi, S. Jaffard and D. Roux, Gaussian processes and pseudodifferential elliptic operators. Rev. Mat. Iberoam. 13 (1997) 19-89.

[3] V. Bentkus, A. Juozulynas and V. Paulauskas, Lévy-LePage series representation of stable vectors: convergence in variation. J. Theoret. Probab. 14 (2001) 949-978.

[4] K.J. Falconer, Tangent fields and the local structure of random fields. J. Theoret. Probab. 15 (2002) 731-750.

[5] K.J. Falconer, The local structure of random processes. J. London Math. Soc. 267 (2003) 657-672.

[6] K.J. Falconer and J. Lévy Véhel, Multifractional, multistable, and other processes with prescribed local form. J. Theoret. Probab. (2008) DOI: 10.1007/s10959-008-0147-9.

[7] K.J. Falconer and L. Lining, Multistable random measures and multistable processes. Preprint (2009).

[8] K.J. Falconer, R. Le Guével and J. Lévy Véhel, Localisable moving average stable and multistable processes. Stoch. Models (2009) 648-672.

[9] T.S. Ferguson and M.J. Klass, A representation of independent increment processes without Gaussian components. Ann. Math. Stat. 43 (1972) 1634-1643.

[10] E. Herbin, From $N$-parameter fractional Brownian motions to $N$-parameter multifractional Brownian motion. Rocky $M t$. J. Math. 36 (2006) 1249-1284.

[11] E. Herbin and J. Lévy Véhel, Stochastic 2 micro-local analysis. Stoch. Proc. Appl. 119 (2009) 2277-2311.

[12] A.N. Kolmogorov, Wienersche Spiralen und einige andere interessante Kurven in Hilbertchen Raume. Doklady 26 (1940) $115-118$.

[13] R. Le Guével and J. Lévy Véhel, A Ferguson-Klass-LePage series representation of multistable multifractional motions and related processes, preprint (2009). Available at http://arxiv.org/abs/0906.5042.

[14] R. Le Page, Multidimensional infinitely divisible variables and processes. I. Stable case Tech. Rep. 292, Dept. Stat., Stanford Univ. (1980).

[15] R. Le Page, Multidimensional infinitely divisible variables and processes, II Probability in Banach Spaces III. Springer, New York, Lect. Notes Math. 860 (1980) 279-284.

[16] M. Ledoux and M. Talagrand, Probability in Banach spaces. Springer-Verlag (1996).

[17] B.B. Mandelbrot and J. Van Ness, Fractional Brownian motion, fractional noises and applications. SIAM Review 10 (1968) 422-437. 
[18] R.F. Peltier and J. Lévy Véhel, Multifractional Brownian motion: definition and preliminary results. Rapport de recherche de l'INRIA, No. 2645 (1995). Available at: http://www-rocq1.inria.fr/fractales/index.php?page=publications.

[19] V. Petrov, Limit Theorems of Probability Theory. Oxford Science Publication (1995).

[20] J. Rosinski, On series representations of infinitely divisible random vectors. Ann. Probab. 18 (1990) 405-430.

[21] G. Samorodnitsky and M.S. Taqqu, Stable Non-Gaussian Random Processes. Chapman and Hall (1994).

[22] S. Stoev and M.S. Taqqu, Stochastic properties of the linear multifractional stable motion. Adv. Appl. Probab. 36 (2004) $1085-1115$

[23] S. Stoev and M.S. Taqqu, Path properties of the linear multifractional stable motion. Fractals 13 (2005) $157-178$.

[24] B. Von Bahr and C.G. Essen, Inequalities for the $r$ th absolute moment of a sum of Random variables, $1 \mathrm{i}=\mathrm{r} i=2$. Ann. Math. Stat. 36 (1965) 299-303. 